\title{
Role of human assets in measuring firm performance and its implication for firm valuation
}

\author{
Moinak Maiti ${ }^{{ }^{*+}+}$ and Darko Vuković and $^{1,2+}$
}

${ }^{*}$ Correspondence:
mmaiti@hse.ru
${ }^{+}$Moinak Maiti and Darko
Vuković contributed equally
to this work
${ }^{1}$ Department for Finance,
St. Petersburg
School of Economics
and Management, National
Research University Higher
School of Economics,
Kantemirovskaya St. 3A, Saint
Petersburg 194100, Russia
Full list of author information
is available at the end of the
article

${ }^{*}$ Correspondence:

mmaiti@hse.ru

Vuković contributed equally

to this work

and Management, National

Research University Higher

school of Economics,

Full list of author information

article

Springer Open

Q The Author(s) 2020 This article is licensed under a Creative Commons Attribution 4.0 International License, which permits use, sharing adaptation, distribution and reproduction in any medium or format, as long as you give appropriate credit to the original author(s) and the source, provide a link to the Creative Commons licence, and indicate if changes were made. The images or other third party material in this article are included in the article's Creative Commons licence, unless indicated otherwise in a credit line to the material. If material is not included in the article's Creative Commons licence and your intended use is not permitted by statutory regulation or exceeds the permitted use, you will need to obtain permission directly from the copyright holder. To view a copy of this licence, visit http://creativeco mmons.org/licenses/by/4.0/.

\section{Abstract}

The purpose of the study is to evaluate the role of human asset in firm performance and its implication for firm valuation. To do so a modified five-factor model with human asset designed for capturing the size, value, profitability and investment in average portfolio returns that performs better than both Fama-French (1993) threeand Fama-French (2015) five-factor model. Study redefines CMA factors as CvMAv that includes human assets in it. The main problem with the modified five-factor model with human asset is the microcap with conservative investment stocks whose returns behave like that low-value unprofitable firms.

Keywords: Human asset, Factor models, Asset Pricing, Risk

JEL Classification: G12

\section{Introduction}

Knowledge-based economy gained momentum over manufacturing-based economy in last few decades. Business firms nowadays specially giving significant importance to develop their human assets to gain competitive advantages over the other firms. Human assets are intangible in nature and generally not captured by balance sheet items. Accurate measurement of firm's human assets is bit complex; one reason could be as Bontis et al. (2000) pointed out that human assets of a firm are not under direct control of the firm. In spite of knowing the fact that human assets are important in valuing the firm still most of the firm valuation are done considering only the balance sheet items. Collins et al. (1997) added investors could gain additional by gathering information on the firms' human assets. Last few decades are spectator of several risk factors and factor models for firm valuations [see Maiti (2020a, b)]. CAPM [Sharpe (1964), Lintner (1965) and Mossin (1966)] was the seminal model that challenged over the period of time by several researchers like Fisher Black (1972), Fama and MacBeth (1973), Ross (1976), Banz (1981), Reinganum (1981), Gibbons (1982), Basu (1983), Shanken (1985) and Bhandari (1988). There after several multifactor models developed and challenged such as Ross (1976) APT model, Fama-French (1993) three-factor model, Fama-French (2015) five-factor model and others. Further studies by Haugen and Baker (1996), Cohen 
et al. (2002), Fairfield et al. (2003), Titman et al. (2004), Novy-Marx (2013), Hou et al. (2014), Clarke (2016), Chiah et al. (2016), Balakrishnan and Maiti (2017), Maiti and Balakrishnan (2018, 2020), Maiti (2019a, b, c), Maiti (2020a, b) and others find that these model are not global and there is a scope for more robust valuation model.

Most of the studies discussed above do not include a measure for human assets in their valuation models. Notably it was the Campbell $(1992,1992)$ studies that strongly argued firm valuation excluding human assets may lead to serious errors. Other studies by Fama and Schwert (1977), Jagannathan and Wang (1996), Jagannathan et al. (1998), Rosett (2001) and Qin (2002) also find that human asset is an important factor in explaining the cross-sectional risk return variations. The present study will examine the role of human asset investment in firm valuation in Indian context. Present study will use Fama-French (1993) three-factor and Fama-French (2015) five-factor model in a time series setup to test the risk return relationship and finally results will be compared to newly proposed model to justify its robustness than the former two models. The nobleness of the present study lies in several ways first altogether a new robust five-factor asset pricing model developed with human asset investment which found to be superior than existing valuation models; second, in Indian context human asset investment factor is almost untouched by previous studies except Maiti (2019a, b, c), Maiti and Balakrishnan (2018, 2020) and few others.

\section{Literature review}

Campbell (1996) shows that by ignoring human asset CAPM overstates the risk investing in financial assets and understates the risk aversion coefficient. Study also finds intertemporal model using human asset to be more robust than the traditional CAPM in explaining risk return relationship. Similarly, Jagannathan and Wang (1996) study also find that the intertemporal model with human asset is more sustainable than CAPM. The study also argues that human assets are tradable such as in mortgage and life insurance markets. Thereafter Rosett (2001) and Qin (2002) added that human asset is positively related to the equity returns. Chen et al. (2005) study find that during the period between 1977 and 2001, there is significant increase in the gap between the book value and market value of the US companies. The study mentioned that ignorance of valuable assets by the financial statement could be the reason behind the gap between the book value and market value as similar to the Collins et al. (1997) findings. The study also confirms that $80 \%$ of the firm's market value is missing in the financial statement of the firm. Knowledge-based aspect of the firms' human asset is considered by Crook et al. (2011). Study argues that human asset characteristics are unique for each firm; human asset of a firm is difficult to copy, replicate and duplicate. Hence, a firm could gain sustainable competitive advantages over other firms by developing its human asset.

In general successful firms pay higher compensation to develop human assets that in turn leads to higher productivity and increase value of the firm. Pantzalis and Park (2009), Edmans (2011) and others study show empirically that market is often unable to price the human asset accurately especially for the small-size firms. From the above discussion it is clear that human asset factor is important in relationship to determining the firm valuation. The main problems lie with human asset is that it is very difficult to measure and none of the available techniques that are used by previous study are able to 
measure the human asset factor accurately. Human asset investment seems to be one of the very important factors that are related to the stock returns and on the other hand, very limited number of studies are done on human asset in this aspects. Considering the emerging markets, very limited number of studies are done on human asset and firm valuation in Indian context. All such factors justify the need of present study and study results will have serious implication for the other emerging and developed markets.

\section{Data and methodology}

\subsection{Data}

The study uses monthly data for total 431 companies those are listed in the BSE 500 index and the study period is from July, 2003 to November, 2016. Study uses Market capitalization $(\mathrm{MC})$ as proxy for Size; Price to Book $(P / B)$ ratio as proxy for Value; BSE 200 index monthly return as proxy for Market $\left(R_{\mathrm{m}}\right)$ and 91-day T-Bills return as proxy for risk-free rate $\left(R_{\mathrm{f}}\right)$. Total asset growth (TA) without human asset act as the proxy for Investment $(\mathrm{CMA})$ and it is calculated by the formula $\left.\left[\left(\mathrm{TA}_{t}-\mathrm{TA}_{t-1}\right) / \mathrm{TA}_{t-1}\right)\right]$ as similar to Hou et al. (2015) and return on equity (ROE) used as the proxy for profitability as similar to Haugen and Baker (1996). Total salary and wage expense used as the proxy for human asset (HA) as similar to Hansson (2004), Draca et al. (2011), and Bell and Machin (2016). Traditionally investment in human asset considers as the cost to the company and not as the investment Petty and Guthrie (2000). But significant number of studies by Bontis (2003) and Wright et al. (2001) argued that human asset should be considered as the investment of the firm rather than expenses in today's knowledge-based economy where human asset has greater importance in determining the value of the firm. So present study defined a new factor for investment with human asset (CvMAv) as the total asset growth (TAM $=\mathrm{TA}+\mathrm{HA})$ including human asset that acts as the proxy for Investment factor $(\mathrm{CvMAv})$ and it is calculated by the formula $\left[\left\{(\mathrm{TA}+\mathrm{HA})_{t}-(\mathrm{TA}+\mathrm{HA})_{t-1}\right\} /\right.$ $\left.\left.(\mathrm{TA}+\mathrm{HA})_{t-1}\right)\right]$.

\subsection{Portfolio construction methodology}

Single and double shorting techniques are deployed to construct the study and mimicking portfolios. Every year in the month of June $(t)$ based on market capitalization of the stocks using single sorting technique five equal weighted portfolios are constructed. Five market capitalization (MC)-based portfolios are named P1 to P5 in ascending order of size. Then again in the month of June next year $(t+1)$ the rank revised using the same process and continued every year till 2016. Following the same procedure other portfolios based on $P / B$, ROE, TA and TAM are constructed and named. Then every year in the month of June $(t)$ using double sorting technique 25 value weighted portfolios are constructed from the cross of five $\mathrm{MC}$ and $P / B$ sorted portfolios. Portfolio consists of small size (MC) and low $P / B$ stocks named as the MP11, similarly portfolio with big size (MC) and high $P / B$ stocks named as MP55. Then again in the month of June next year $(t+1)$ the rank revised using the same process and continued every year till 2016. Following the same procedure using double sorting technique three sets of 25 portfolios are constructed from the each individual crosses of five 'MC \& ROE', 'MC \& TA' and 'MC \& TAM' sorted portfolios, respectively. The portfolios were named in the similar fashion as 
explained in case of 'MC \& $P / B$ ' cross and again in the month of June next year $(t+1)$ the rank revised using the same process and continued every year till 2016.

Using the similar process, other portfolios are constructed to derive the mimicking portfolios as described below. Every year in the month of June $(t)$ based on market capitalization $(\mathrm{MC})$ of the stocks using single sorting technique two equal weighted portfolios are constructed similar to Balakrishnan and Maiti (2017), Maiti and Balakrishnan (2018, 2020), Maiti (2019a, b, c) and other studies. The portfolios are named Small (S) and Big (B) in ascending order of size (MC). Then again in the month of June next year $(t+1)$ the rank revised using the same process and continued every year till 2016. Then using Fama-French (1993) breakpoints (30:40:30) based on $P / B$ three weighted portfolios are constructed. Portfolio consists of bottom $30 \%$ stocks with low $P / B$ ratio named value $(V)$, top $30 \%$ stocks with high $P / B$ ratio named growth $(G)$ and rest $40 \%$ stocks were placed into the neutral portfolio. Then again in the month of June next year $(t+1)$ the rank revised using the same process and continued every year till 2016. Then every year in the month of June $(t)$ using double sorting technique six value weighted portfolios are constructed from the cross of two MC and three $P / B$ sorted portfolios. These formed six portfolios are named as $S / L, S / N, S / G, B / L, B / N$ and $B / G$, where $S / L$ consists of small size and low $P / B$ stocks whereas $B / G$ consists of big size and high $P / B$ stocks. Similarly using Fama-French (1993) breakpoints (30:40:30) based on ROE, TA and TAM three sets of three weighted portfolios are constructed from each of these variables. Portfolio consists of bottom 30\% stocks with low ROE named Weak (W), top 30\% stocks with high ROE named Robust (R) and rest 40\% stocks were placed into the neutral portfolio. Then portfolio consists of bottom 30\% stocks with low TA named Conservative $(C)$, top 30\% stocks with high TA named Aggressive $(A)$ and rest $40 \%$ stocks were placed into the neutral portfolio. Similarly portfolio consists of bottom $30 \%$ stocks with low TAM named Conservative Value (CV), top 30\% stocks with high TAM named Aggressive value (AV) and rest $40 \%$ stocks were placed into the neutral portfolio. Then again in the month of June next year $(t+1)$ the rank revised using the same process and continued every year till 2016. Then every year in the month of June $(t)$ using double sorting technique three sets of six value weighted portfolios are constructed from the each individual cross of 'two MC \& three ROE', 'two MC \& three TA' and 'two MC \& three TAM' sorted portfolios, respectively. Then formed portfolios were named in similar fashion as described in case of 'two MC \& three ROE' cross.

\subsection{Mimicking portfolios}

Study uses five mimicking portfolios SMB (Size), LMH (value), RMW (profitability), CMA (Investment without human asset) and CvMAv (Investment with human asset) and they are calculated as explained below:

$$
\begin{aligned}
& \mathrm{SMB}=(S / L+S / M+S / H) / 3-(B / L+B / M+B / H) / 3, \\
& \mathrm{LMH}=(S / L+B / L) / 2-(S / H+B / H) / 2, \\
& \mathrm{RMW}=(S / R+B / R) / 2-(S / W+B / W) / 2,
\end{aligned}
$$




$$
\begin{aligned}
& \mathrm{CMA}=(S / C+B / C) / 2-(S / A+B / A) / 2, \\
& \mathrm{CvMAv}=(S / \mathrm{Cv}+B / \mathrm{Cv}) / 2-(S / \mathrm{Av}+B / \mathrm{Av}) / 2 .
\end{aligned}
$$

The present study uses three regression models:

Fama-French three-factor model

$$
R_{\mathrm{Pt}}-R_{\mathrm{Ft}}=a+b\left(R_{\mathrm{Mt}}-R_{\mathrm{Ft}}\right)+s \mathrm{SMB}_{t}+l \mathrm{LMH}_{t}+e_{t}
$$

where SMB and LMH mimic the risk factors whereas s and 1 are the portfolio's responsiveness to (sensitivity coefficients) SMB and LMH factors, respectively.

Fama-French Five-factor model

$$
R_{\mathrm{Pt}}-R_{\mathrm{Ft}}=a+b\left(R_{\mathrm{Mt}}-R_{\mathrm{Ft}}\right)+s \mathrm{SMB}_{t}+l \mathrm{LMH}_{t}+p \mathrm{RMWt}+t \mathrm{CMAt}+e_{t}
$$

where SMB, LMH, RMW and CMA mimic the risk factors whereas s, l, p and t are the portfolio's responsiveness to (sensitivity coefficients) SMB, LMH, RMW and CMA factors, respectively.

Modified five-factor model with Human Asset

$$
R_{\mathrm{Pt}}-R_{\mathrm{Ft}}=a+b\left(R_{\mathrm{Mt}}-R_{\mathrm{Ft}}\right)+s \mathrm{SMB}_{t}+l \mathrm{LMH}_{t}+p \mathrm{RMWt}+h \mathrm{CvMAvt}+e_{t}
$$

where SMB, LMH, RMW and CvMAv mimic the risk factors whereas $\mathrm{s}, \mathrm{l}, \mathrm{p}$ and $\mathrm{t}$ are the portfolio's responsiveness to (sensitivity coefficients) SMB, LMH, RMW and CvMAv factors, respectively.

\section{Explanatory variables}

Descriptive statistics of the explanatory variables are shown in Table 1 . The average monthly market premium of $1 \%(t=2.151)$, size premium of $1.3 \%(t=5.177)$ and value premium of $0.8 \%(t=2.164)$ seem quite attractive in terms of investment; results are similar to Fama-French $(1993,2015)$ in US market. Investment based on size of the firms has higher probability to yield more returns to the investors. Profitability, investment and human asset factor yields comparatively much lower average monthly premium than market, size and value factor. That implies that risks associated with these factors are lower and risk adverse investors should consider these factors while making investment decision.

Correlation matrix for the explanatory variables is shown in Table 2. Market is negatively related to the profitability and investment factors whereas positively related to size, value and investment with human asset factors. Theoretically findings are correct and similar to Fama-French (2015) in US context. Value and investment with human

Table 1 Descriptive statistics for the independent variables

\begin{tabular}{lllllll}
\hline & Rm & SMB & LMH & RMW & CMA & CvMAv \\
\hline Mean returns & 0.010 & 0.013 & 0.008 & 0.001 & 0.001 & 0.002 \\
Standard deviations & 0.059 & 0.031 & 0.047 & 0.006 & 0.028 & 0.012 \\
T-statistics & 2.151 & 5.177 & 2.164 & 2.115 & 0.617 & 2.115 \\
\hline
\end{tabular}


Table 2 Correlation matrix for explanatory variables

\begin{tabular}{lllllll}
\hline & RM & SMB & LMH & RMW & CvMAv & CMA \\
\hline RM & 1 & & & & & \\
SMB & 0.283 & 1 & & & & \\
LMH & 0.329 & 0.063 & 1 & & & \\
RMW & -0.352 & -0.041 & -0.843 & 1 & 1 \\
CVMAV & 0.306 & 0.287 & 0.171 & -0.338 & 0.162 & 1 \\
CMA & -0.133 & 0.286 & 0.215 & -0.346 & \\
\hline
\end{tabular}

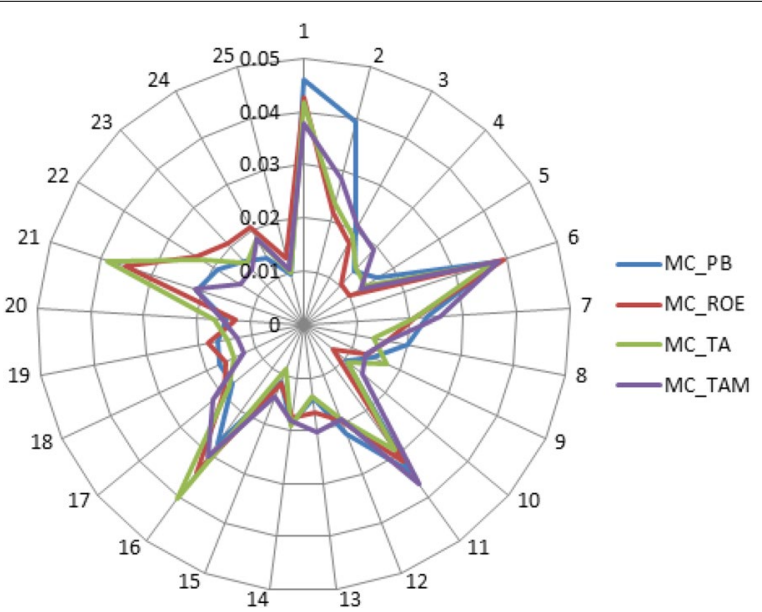

Fig. 1 Radar graph shows 25 portfolios mean returns based on MC_PB, MC_ROE, MC_TA and MC_TAM crosses

asset factors are positively related to other factors except profitability factor. The correlations between the explanatory variables are within the limit and in all cases standard errors are less.

\section{Empirical results}

Figure 1 shows the average return pattern for 25 portfolios constructed based on MC_ PB, MC_ROE, MC_TA and MC_TAM. In all the cases portfolio 1 returns outperformed the returns from other portfolios and similarly portfolio 25 has the lowest average returns in all the cases. The return patterns clearly reveal that investors can gain abnormal returns by following size-based investment strategy. Figure 1 also reveals that there is manifestation of other factors (value, profitability, investment) too and there exists certain patterns which need to be identified.

Details of each portfolio formed from each cross are shown in Table 3 for further discussion.

MC_PB: First portfolio consists of small size and low $P / B$ stocks yield average monthly return of $4.6 \%(t=5.490)$ which is at least four times higher than the average monthly return of last portfolio. Holding value $(P / B)$ constant in each column the average monthly return decreases with increase in size and this is known as the size effect. Consider first column keeping low-value $(P / B)$ constant first portfolio at the top of 
Table 3 Summary statistics of 25 portfolios formed on MC_PB, MC_ROE, MC_TA and MC TAM

\begin{tabular}{|c|c|c|c|c|c|c|c|c|c|c|}
\hline \multicolumn{11}{|c|}{ Panel A (mean excess returns) } \\
\hline & \multicolumn{5}{|c|}{ MC_PB } & \multicolumn{5}{|c|}{ MC_ROE } \\
\hline & Low & 2 & 3 & 4 & High & Low & 2 & 3 & 4 & High \\
\hline Small & 0.046 & 0.039 & 0.034 & 0.027 & 0.021 & 0.043 & 0.039 & 0.032 & 0.034 & 0.035 \\
\hline 2 & 0.039 & 0.023 & 0.022 & 0.017 & 0.019 & 0.022 & 0.021 & 0.019 & 0.019 & 0.024 \\
\hline 3 & 0.020 & 0.020 & 0.014 & 0.017 & 0.016 & 0.018 & 0.016 & 0.017 & 0.016 & 0.021 \\
\hline 4 & 0.014 & 0.014 & 0.019 & 0.016 & 0.014 & 0.010 & 0.013 & 0.018 & 0.018 & 0.021 \\
\hline \multirow[t]{3}{*}{ Big } & 0.016 & 0.010 & 0.011 & 0.015 & 0.010 & 0.010 & 0.007 & 0.012 & 0.013 & 0.013 \\
\hline & \multicolumn{5}{|c|}{ MC_TA } & \multicolumn{5}{|c|}{ MC_TAM } \\
\hline & Low & 2 & 3 & 4 & High & Low & 2 & 3 & 4 & High \\
\hline Small & 0.042 & 0.037 & 0.029 & 0.040 & 0.039 & 0.038 & 0.039 & 0.037 & 0.030 & 0.022 \\
\hline 2 & 0.024 & 0.021 & 0.019 & 0.018 & 0.023 & 0.028 & 0.026 & 0.019 & 0.022 & 0.014 \\
\hline 3 & 0.019 & 0.013 & 0.013 & 0.014 & 0.016 & 0.021 & 0.016 & 0.020 & 0.012 & 0.014 \\
\hline 4 & 0.014 & 0.017 & 0.019 & 0.015 & 0.018 & 0.019 & 0.013 & 0.018 & 0.013 & 0.019 \\
\hline Big & 0.014 & 0.011 & 0.009 & 0.017 & 0.010 & 0.013 & 0.014 & 0.014 & 0.015 & 0.011 \\
\hline
\end{tabular}

Panel B (standard deviations)

\begin{tabular}{|c|c|c|c|c|c|c|c|c|c|c|}
\hline & \multicolumn{5}{|c|}{ MC_PB } & \multicolumn{5}{|c|}{ MC_ROE } \\
\hline & Low & 2 & 3 & 4 & High & Low & 2 & 3 & 4 & High \\
\hline Small & 0.106 & 0.101 & 0.093 & 0.083 & 0.087 & 0.109 & 0.164 & 0.095 & 0.090 & 0.091 \\
\hline 2 & 0.149 & 0.100 & 0.084 & 0.084 & 0.078 & 0.111 & 0.099 & 0.086 & 0.086 & 0.083 \\
\hline 3 & 0.109 & 0.104 & 0.082 & 0.088 & 0.076 & 0.112 & 0.101 & 0.092 & 0.086 & 0.082 \\
\hline 4 & 0.116 & 0.097 & 0.108 & 0.089 & 0.067 & 0.110 & 0.100 & 0.089 & 0.084 & 0.069 \\
\hline \multirow[t]{3}{*}{ Big } & 0.108 & 0.104 & 0.087 & 0.089 & 0.067 & 0.103 & 0.100 & 0.086 & 0.080 & 0.078 \\
\hline & \multicolumn{5}{|c|}{ MC_TA } & \multicolumn{5}{|c|}{ MC_TAM } \\
\hline & Low & 2 & 3 & 4 & High & Low & 2 & 3 & 4 & High \\
\hline Small & 0.103 & 0.107 & 0.099 & 0.145 & 0.104 & 0.110 & 0.099 & 0.141 & 0.092 & 0.087 \\
\hline 2 & 0.099 & 0.094 & 0.088 & 0.085 & 0.096 & 0.112 & 0.093 & 0.088 & 0.088 & 0.085 \\
\hline 3 & 0.096 & 0.088 & 0.093 & 0.093 & 0.101 & 0.103 & 0.091 & 0.087 & 0.101 & 0.083 \\
\hline 4 & 0.093 & 0.089 & 0.088 & 0.087 & 0.111 & 0.101 & 0.079 & 0.084 & 0.089 & 0.075 \\
\hline Big & 0.082 & 0.074 & 0.080 & 0.086 & 0.094 & 0.092 & 0.104 & 0.089 & 0.082 & 0.077 \\
\hline \multicolumn{11}{|c|}{ Panel C ( $T$-statistics) } \\
\hline & \multicolumn{5}{|c|}{ MC_PB } & \multicolumn{5}{|c|}{ MC_ROE } \\
\hline & Low & 2 & 3 & 4 & High & Low & 2 & 3 & 4 & High \\
\hline Small & 5.490 & 4.843 & 4.615 & 4.187 & 3.086 & 4.953 & 3.043 & 4.228 & 4.803 & 4.902 \\
\hline 2 & 3.324 & 2.961 & 3.330 & 2.633 & 3.101 & 2.465 & 2.673 & 2.854 & 2.873 & 3.610 \\
\hline 3 & 2.347 & 2.436 & 2.159 & 2.508 & 2.742 & 1.991 & 1.987 & 2.299 & 2.405 & 3.241 \\
\hline 4 & 1.510 & 1.851 & 2.204 & 2.327 & 2.718 & 1.195 & 1.650 & 2.502 & 2.774 & 3.851 \\
\hline \multirow[t]{3}{*}{ Big } & 1.927 & 1.267 & 1.659 & 2.093 & 1.841 & 1.233 & 0.921 & 1.719 & 2.017 & 2.117 \\
\hline & \multicolumn{5}{|c|}{ MC_TA } & \multicolumn{5}{|c|}{ MC_TAM } \\
\hline & Low & 2 & 3 & 4 & High & Low & 2 & 3 & 4 & High \\
\hline Small & 5.123 & 4.425 & 3.736 & 3.538 & 4.708 & 4.344 & 4.946 & 3.304 & 4.186 & 3.138 \\
\hline 2 & 3.022 & 2.800 & 2.755 & 2.701 & 2.982 & 3.187 & 3.474 & 2.719 & 3.189 & 2.076 \\
\hline 3 & 2.507 & 1.942 & 1.848 & 1.972 & 1.980 & 2.595 & 2.236 & 2.934 & 1.569 & 2.140 \\
\hline
\end{tabular}


Table 3 (continued)

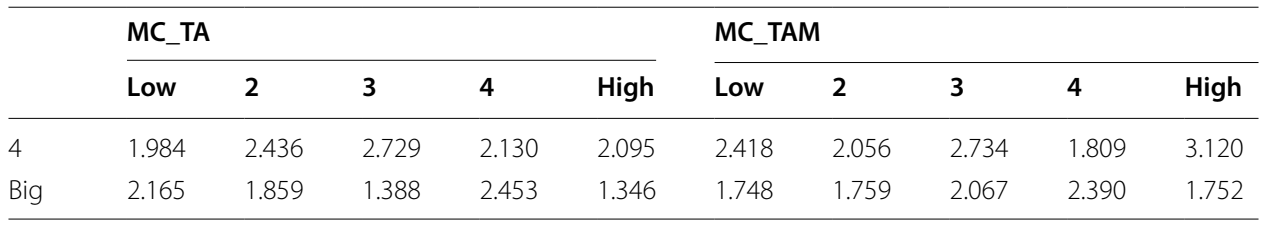

column with small-size stocks' portfolio yields average monthly return of 4.6\% ( $t=5.490)$ whereas average monthly return reduced more than thrice to $1.6 \%(t=1.927)$ for the bottom portfolio in the column with big-size stocks. Same observation is observed for all other columns that indicate there is a strong size effect in portfolio return patterns. Similarly holding size (MC) constant in each row the average monthly return decreases with increase in value and this is known as the value effect. Consider first row keeping small-size constant first portfolio at the extreme left of the first row with low-value stocks' portfolio yields average monthly return of $4.6 \%(t=5.490)$ whereas average monthly return reduced almost half to $2.1 \%(t=3.086)$ for the bottom portfolio in the column with big-size stocks. Similar observation reflects in all other rows also indicate that there is a strong value effect in portfolio return patterns. The study findings are similar to Fama-French (2015) study in US context.

MC_ROE, MC_TA and MC_TAM: Clear size effect is observed in portfolios formed from MC_ROE, MC_TA and MC_TAM crossed portfolios. In MC_ROE cross portfolio holding size constant for first three rows shows no clear pattern but last two rows with big-size portfolios show weak profitability effect as similar to Novy-Marx (2013) and Fama-French (2015) findings. Holding size constant for both the MC_TA and MC_ TAM crosses no much clear portfolio return pattern observed while traversing through any of the other rows.

Figure 2 shows the 3D view of return patterns for all the portfolios formed from MC_PB, MC_ROE, MC_TA and MC_TAM crosses, respectively. More complex return patterns are observed in case for MC_ROE, MC_TA and MC_TAM cross portfolios as frequent change in slope can be observed in Fig. 2.

\section{Asset pricing results}

\subsection{Three-factor regression}

Three-factor regression results with market, size and value are shown in Table 4. A regression model is said to be a best model which is able to capture all alpha values equal to zero. Five portfolios found significant (alpha values not equal to zero where $t(\mathrm{a})$ is more than 1.96) for MC_PB, MC_ROE \& MC_TA and four portfolios found significant in case of MC_TAM portfolios. Average alpha value (intercepts) for MC_PB, MC_ROE, MC_TA and MC_TAM cross portfolios found to be $0.0034,0.0049,0.0034$ and 0.0033 , respectively. The average values of intercepts are not closer to zero for all the cases and average R-Square value (\%) for MC_PB, MC_ROE, MC_TA and MC_TAM cross portfolios are 78.7, 76.4, 76.6 and 73.8, respectively (see Table 7). Expect for the MC_TAM cross portfolio consists of microcaps (First portfolio) that are not captured by the threefactor regressions. The study findings are similar to the global findings of Aharoni et al. (2013) and Fama-French (2015). 

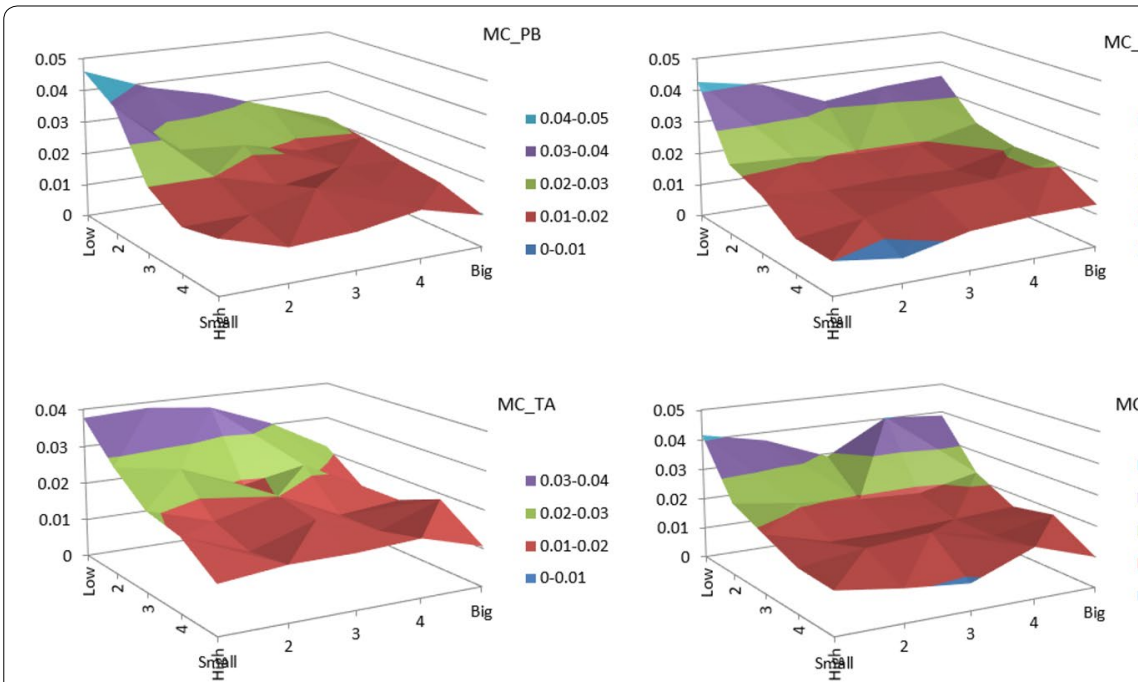

MC_ROE

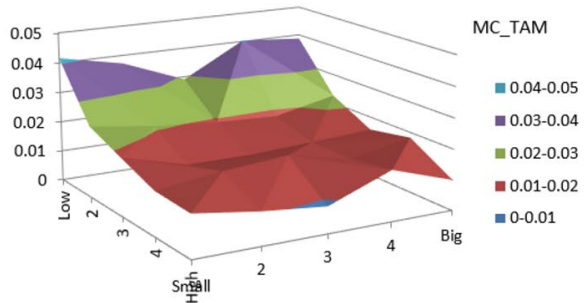

Fig. 2 Visual 3D surface images for 25 portfolios mean returns formed on MC_PB, MC_ROE, MC_TA and MC_TAM

Microcaps remain the problem for most of the crosses expect MC_TAM cross. Since three-factor model is unable to explain all risk return relationship, this study further estimates five-factor regression with market, size, value, profitability and investment (without human investment).

\subsection{Five-factor regressions (without human asset investment)}

Five-factor regression results with market, size; value, profitability and investment (without human investment) are shown in Table 5.

MC_PB: Five portfolios found to be significant with average alpha value of 0.0047 and 79.9 average $R$-square (\%). Market and value slopes found to be highly positive, size slopes are positive for small-size stock portfolio but becomes slightly negative toward big-size portfolios. Five factors provide more information than three-factor model on the risk return relationship for the first portfolio with microcaps. In column one with low-value stocks, the coefficient of profitability (RMW) factor has positive slopes for microcaps whereas it changes its sign on increase of size. This indicates that first portfolio with combination of low value and microcaps is much dominated by the low-value stocks with aggressive investment and marginal profitability but yields higher returns than other portfolios as shown in Table 3 . Value (LMH) coefficients are highly positive for low $P / B$ stock portfolio and become negative for high $P / B$ stock portfolios. MC_PB cross is not based on the investment factor but it seems that results are aligned with it as low $P / B$ stock portfolios show lower negative investment (CMA) than high $P / B$ stock portfolios. Theoretically, it justifies as low $P / B$ firms are conservative investment nature whereas high $P / B$ firms have aggressive investment nature.

MC_ROE: Five portfolios found to be significant with average alpha value of 0.0048 and 78.2 average $R$-square (\%). Profitability (RMW) and investment (CMA) coefficient changes its sign on moving from low ROE stock portfolios to the high ROE stock portfolios. High positive investment (CMA) and less negative profitability (RMW) coefficient 
Table $4 \mathrm{FF}$ three-factor regression results $R_{\mathrm{Pt}}-R_{\mathrm{Ft}}=a+b\left(R_{\mathrm{Mt}}-R_{\mathrm{Ft}}\right)+s \mathrm{SMB}_{t}+I \mathrm{LMH}_{t}+e_{t}$

Panel A (MC_PB

\begin{tabular}{|c|c|c|c|c|c|c|c|c|c|c|}
\hline & \multicolumn{5}{|l|}{$a$} & \multicolumn{5}{|l|}{ RM } \\
\hline & Low & 2 & 3 & 4 & High & Low & 2 & 3 & 4 & High \\
\hline Small & 0.013 & 0.009 & 0.009 & 0.002 & -0.004 & 1.038 & 1.093 & 1.103 & 1.011 & 1.096 \\
\hline 2 & 0.001 & -0.001 & 0.000 & -0.005 & 0.002 & 0.804 & 1.075 & 0.951 & 1.021 & 1.019 \\
\hline 3 & -0.006 & -0.003 & -0.003 & 0.001 & 0.003 & 1.118 & 1.199 & 0.920 & 1.139 & 0.979 \\
\hline 4 & -0.003 & 0.001 & 0.006 & 0.002 & 0.004 & 1.050 & 0.952 & 1.258 & 1.139 & 0.866 \\
\hline \multirow[t]{3}{*}{ Big } & 0.003 & 0.000 & 0.002 & 0.002 & 0.001 & 0.994 & 1.106 & 1.024 & 1.139 & 0.911 \\
\hline & \multicolumn{5}{|l|}{$S$} & \multicolumn{5}{|l|}{$L$} \\
\hline & Low & 2 & 3 & 4 & High & Low & 2 & 3 & 4 & High \\
\hline Small & 1.423 & 1.220 & 0.967 & 1.193 & 1.231 & 0.631 & 0.414 & 0.183 & 0.062 & -0.165 \\
\hline 2 & 1.464 & 0.772 & 0.795 & 0.893 & 0.629 & 1.433 & 0.427 & 0.282 & 0.148 & -0.152 \\
\hline 3 & 0.680 & 0.575 & 0.415 & 0.471 & 0.358 & 0.813 & 0.470 & 0.295 & -0.064 & -0.115 \\
\hline 4 & -0.184 & -0.096 & -0.135 & 0.270 & 0.248 & 1.121 & 0.666 & 0.247 & -0.064 & -0.227 \\
\hline \multirow[t]{3}{*}{ Big } & -0.361 & -0.401 & -0.287 & 0.270 & 0.181 & 0.998 & 0.628 & 0.303 & -0.064 & -0.270 \\
\hline & \multicolumn{5}{|l|}{$t(a)$} & \multicolumn{5}{|l|}{$t(\mathrm{RM})$} \\
\hline & Low & 2 & 3 & 4 & High & Low & 2 & 3 & 4 & High \\
\hline Small & 2.601 & 1.966 & 2.262 & 0.522 & -1.119 & 15.455 & 17.404 & 19.464 & 21.447 & 21.491 \\
\hline 2 & 0.135 & -0.113 & 0.061 & -1.560 & 0.683 & 6.286 & 16.486 & 18.565 & 21.907 & 22.855 \\
\hline 3 & -1.979 & -0.774 & -0.763 & 0.156 & 0.984 & 19.763 & 22.420 & 18.153 & 25.087 & 23.376 \\
\hline 4 & -0.821 & 0.155 & 1.987 & 0.611 & 1.536 & 19.386 & 16.925 & 21.962 & 23.993 & 21.939 \\
\hline \multirow[t]{3}{*}{ Big } & 0.928 & -0.158 & 1.047 & 0.611 & 0.239 & 21.391 & 25.439 & 31.552 & 23.993 & 28.252 \\
\hline & \multicolumn{5}{|l|}{$t(s)$} & \multicolumn{5}{|l|}{$t(I)$} \\
\hline & Low & 2 & 3 & 4 & High & Low & 2 & 3 & 4 & High \\
\hline Small & 9.517 & 8.727 & 7.670 & 11.378 & 10.844 & 6.230 & 4.366 & 2.147 & 0.874 & -2.151 \\
\hline 2 & 5.142 & 5.321 & 6.977 & 8.615 & 6.335 & 7.425 & 4.345 & 3.651 & 2.110 & -2.259 \\
\hline 3 & 5.400 & 4.834 & 3.679 & 4.659 & 3.841 & 9.533 & 5.830 & 3.862 & -0.940 & -1.820 \\
\hline 4 & -1.528 & -0.769 & -1.058 & 2.552 & 2.820 & 13.720 & 7.857 & 2.861 & -0.889 & -3.813 \\
\hline Big & -3.496 & -4.147 & -3.978 & 2.552 & 2.527 & 14.238 & 9.581 & 6.192 & -0.889 & -5.559 \\
\hline
\end{tabular}

\section{Panel B MC_ROE}

\begin{tabular}{|c|c|c|c|c|c|c|c|c|c|c|}
\hline & \multicolumn{5}{|l|}{$a$} & \multicolumn{5}{|l|}{ RM } \\
\hline & Low & 2 & 3 & 4 & High & Low & 2 & 3 & 4 & High \\
\hline Small & 0.006 & 0.007 & 0.004 & 0.007 & 0.008 & 1.076 & 0.776 & 0.998 & 1.037 & 1.108 \\
\hline 2 & -0.006 & -0.003 & -0.001 & -0.002 & 0.005 & 1.091 & 1.051 & 1.011 & 1.073 & 1.037 \\
\hline 3 & -0.007 & -0.002 & 0.000 & -0.001 & 0.008 & 1.106 & 1.115 & 1.083 & 0.991 & 1.028 \\
\hline 4 & -0.009 & -0.002 & 0.007 & 0.009 & 0.010 & 1.150 & 0.996 & 0.957 & 0.953 & 0.903 \\
\hline \multirow[t]{3}{*}{ Big } & -0.003 & -0.005 & 0.002 & 0.003 & 0.005 & 1.136 & 1.123 & 1.027 & 1.013 & 1.006 \\
\hline & \multicolumn{5}{|l|}{$S$} & \multicolumn{5}{|l|}{$L$} \\
\hline & Low & 2 & 3 & 4 & High & Low & 2 & 3 & 4 & High \\
\hline Small & 1.667 & 1.189 & 1.094 & 1.175 & 1.351 & 0.617 & 1.251 & 0.457 & 0.210 & -0.110 \\
\hline 2 & 0.858 & 0.669 & 0.658 & 0.925 & 0.629 & 0.808 & 0.562 & 0.183 & -0.068 & 0.006 \\
\hline 3 & 0.618 & 0.232 & 0.294 & 0.421 & 0.197 & 0.764 & 0.451 & 0.244 & 0.270 & -0.004 \\
\hline 4 & 0.214 & -0.043 & -0.163 & -0.099 & 0.300 & 0.611 & 0.680 & 0.360 & 0.130 & -0.200 \\
\hline Big & -0.222 & -0.220 & -0.158 & -0.018 & -0.003 & 0.516 & 0.469 & 0.220 & -0.028 & -0.209 \\
\hline
\end{tabular}


Table 4 (continued)

\begin{tabular}{|c|c|c|c|c|c|c|c|c|c|c|}
\hline & \multicolumn{5}{|l|}{$t(a)$} & \multicolumn{5}{|l|}{$t(\mathrm{RM})$} \\
\hline & Low & 2 & 3 & 4 & High & Low & 2 & 3 & 4 & High \\
\hline Small & 1.997 & 0.566 & 0.979 & 1.831 & 1.916 & 16.349 & 4.765 & 16.617 & 18.954 & 19.143 \\
\hline 2 & -1.330 & -0.620 & -0.151 & -0.660 & 1.491 & 16.180 & 18.117 & 19.342 & 20.701 & 20.755 \\
\hline 3 & -1.476 & -0.405 & 0.051 & -0.308 & 2.597 & 16.007 & 18.809 & 21.125 & 19.774 & 23.729 \\
\hline 4 & -1.775 & -0.416 & 1.968 & 2.433 & 3.518 & 17.190 & 17.404 & 18.116 & 18.402 & 23.898 \\
\hline \multirow[t]{3}{*}{ Big } & -0.739 & -1.501 & 0.622 & 1.103 & 1.628 & 23.714 & 25.233 & 27.717 & 25.631 & 25.179 \\
\hline & \multicolumn{5}{|l|}{$t(s)$} & \multicolumn{5}{|l|}{$t(l)$} \\
\hline & Low & 2 & 3 & 4 & High & Low & 2 & 3 & 4 & High \\
\hline Small & 11.381 & 3.279 & 8.188 & 9.654 & 10.493 & 6.217 & 5.091 & 5.051 & 2.547 & -1.257 \\
\hline 2 & 5.718 & 5.185 & 5.659 & 8.019 & 5.660 & 7.949 & 6.428 & 2.322 & -0.872 & 0.084 \\
\hline 3 & 4.018 & 1.758 & 2.575 & 3.778 & 2.039 & 7.330 & 5.050 & 3.161 & 3.566 & -0.057 \\
\hline 4 & 1.436 & -0.338 & -1.390 & -0.862 & 3.572 & 6.051 & 7.871 & 4.519 & 1.669 & -3.509 \\
\hline Big & -2.080 & -2.220 & -1.915 & -0.202 & -0.039 & 7.136 & 6.984 & 3.938 & -0.476 & -3.473 \\
\hline
\end{tabular}

\section{Panel C MC_TA}

\begin{tabular}{|c|c|c|c|c|c|c|c|c|c|c|}
\hline & \multicolumn{5}{|l|}{$a$} & \multicolumn{5}{|l|}{ RM } \\
\hline & Low & 2 & 3 & 4 & High & Low & 2 & 3 & 4 & High \\
\hline Small & 0.009 & 0.005 & 0.001 & 0.009 & 0.006 & 1.051 & 1.143 & 1.071 & 0.788 & 1.108 \\
\hline 2 & 0.000 & -0.001 & -0.003 & 0.000 & 0.000 & 1.080 & 1.048 & 0.969 & 0.929 & 1.086 \\
\hline 3 & -0.004 & -0.004 & -0.004 & 0.000 & -0.004 & 1.099 & 0.978 & 1.139 & 1.119 & 1.093 \\
\hline 4 & 0.000 & 0.004 & 0.006 & 0.002 & 0.005 & 1.049 & 1.016 & 0.988 & 0.984 & 1.282 \\
\hline \multirow[t]{3}{*}{ Big } & 0.003 & 0.002 & 0.000 & 0.009 & -0.001 & 0.971 & 0.830 & 1.004 & 1.031 & 1.181 \\
\hline & \multicolumn{5}{|l|}{$S$} & \multicolumn{5}{|l|}{$L$} \\
\hline & Low & 2 & 3 & 4 & High & Low & 2 & 3 & 4 & High \\
\hline Small & 1.629 & 1.314 & 1.073 & 1.274 & 1.448 & 0.262 & 0.536 & 0.458 & 0.920 & 0.371 \\
\hline 2 & 0.700 & 0.704 & 0.667 & 0.449 & 0.793 & 0.556 & 0.345 & 0.448 & 0.403 & 0.270 \\
\hline 3 & 0.653 & 0.385 & 0.349 & 0.164 & 0.494 & 0.478 & 0.345 & 0.226 & 0.200 & 0.306 \\
\hline 4 & 0.179 & 0.005 & 0.027 & -0.009 & -0.011 & 0.195 & 0.314 & 0.333 & 0.339 & 0.078 \\
\hline \multirow[t]{3}{*}{ Big } & 0.052 & -0.131 & -0.120 & -0.304 & -0.042 & 0.135 & 0.282 & 0.070 & 0.151 & 0.018 \\
\hline & \multicolumn{5}{|l|}{$t(a)$} & \multicolumn{5}{|l|}{$t(\mathrm{RM})$} \\
\hline & Low & 2 & 3 & 4 & High & Low & 2 & 3 & 4 & High \\
\hline Small & 2.029 & 1.103 & 0.285 & 1.975 & 1.997 & 16.214 & 18.425 & 17.937 & 5.504 & 16.419 \\
\hline 2 & -0.101 & -0.311 & -0.711 & 0.017 & -0.090 & 19.564 & 17.682 & 19.469 & 17.775 & 17.412 \\
\hline 3 & -1.107 & -1.012 & -1.250 & -0.086 & -0.760 & 22.238 & 18.320 & 25.376 & 22.580 & 15.654 \\
\hline 4 & 0.055 & 1.279 & 1.978 & 0.669 & 1.000 & 17.536 & 20.961 & 20.534 & 22.067 & 18.501 \\
\hline \multirow[t]{3}{*}{ Big } & 0.775 & 0.734 & -0.145 & 3.479 & -0.417 & 21.513 & 22.036 & 32.640 & 28.766 & 26.228 \\
\hline & \multicolumn{5}{|l|}{$t(s)$} & \multicolumn{5}{|l|}{$t(l)$} \\
\hline & Low & 2 & 3 & 4 & High & Low & 2 & 3 & 4 & High \\
\hline Small & 10.303 & 9.518 & 8.074 & 4.002 & 9.649 & 2.529 & 5.724 & 5.086 & 4.264 & 3.641 \\
\hline 2 & 5.698 & 5.337 & 6.021 & 3.863 & 5.717 & 6.676 & 3.860 & 5.969 & 5.109 & 2.870 \\
\hline 3 & 5.939 & 3.239 & 3.495 & 1.490 & 3.180 & 6.419 & 4.283 & 3.337 & 2.677 & 2.908 \\
\hline 4 & 1.346 & 0.048 & 0.248 & -0.088 & -0.074 & 2.162 & 4.299 & 4.589 & 5.038 & 0.748 \\
\hline Big & 0.516 & -1.569 & -1.750 & -3.814 & -0.419 & 1.978 & 4.961 & 1.518 & 2.800 & 0.270 \\
\hline
\end{tabular}


Table 4 (continued)

\begin{tabular}{|c|c|c|c|c|c|c|c|c|c|c|}
\hline \multicolumn{11}{|c|}{ Panel D MC_TAM } \\
\hline & \multicolumn{5}{|l|}{$a$} & \multicolumn{5}{|l|}{ RM } \\
\hline & Low & 2 & 3 & 4 & High & Low & 2 & 3 & 4 & High \\
\hline Small & 0.003 & 0.013 & 0.002 & 0.003 & -0.001 & 1.291 & 0.851 & 0.819 & 0.957 & 0.897 \\
\hline 2 & 0.001 & 0.002 & 0.001 & 0.000 & -0.002 & 1.192 & 1.015 & 0.973 & 0.923 & 0.933 \\
\hline 3 & -0.006 & -0.001 & 0.005 & -0.004 & 0.002 & 1.224 & 1.040 & 1.046 & 1.124 & 0.904 \\
\hline 4 & 0.001 & -0.001 & 0.006 & 0.003 & 0.007 & 1.187 & 0.834 & 0.906 & 0.907 & 0.914 \\
\hline \multirow[t]{3}{*}{ Big } & 0.004 & 0.002 & 0.002 & 0.007 & 0.001 & 0.987 & 1.307 & 1.083 & 0.965 & 1.000 \\
\hline & \multicolumn{5}{|l|}{$S$} & \multicolumn{5}{|l|}{$L$} \\
\hline & Low & 2 & 3 & 4 & High & Low & 2 & 3 & 4 & High \\
\hline Small & 1.615 & 0.901 & 1.457 & 1.164 & 0.867 & 0.125 & 0.662 & 0.988 & 0.355 & 0.387 \\
\hline 2 & 0.831 & 0.811 & 0.443 & 0.757 & 0.289 & 0.579 & 0.431 & 0.317 & 0.449 & 0.327 \\
\hline 3 & 1.053 & 0.392 & 0.305 & 0.132 & 0.031 & 0.185 & 0.269 & 0.076 & 0.405 & 0.393 \\
\hline 4 & 0.399 & 0.176 & 0.090 & -0.261 & 0.178 & 0.202 & 0.371 & 0.295 & 0.529 & 0.003 \\
\hline \multirow[t]{3}{*}{ Big } & -0.171 & -0.043 & 0.039 & -0.231 & 0.030 & 0.133 & 0.004 & 0.098 & 0.177 & -0.130 \\
\hline & \multicolumn{5}{|l|}{$t(a)$} & \multicolumn{5}{|l|}{$t(\mathrm{RM})$} \\
\hline & Low & 2 & 3 & 4 & High & Low & 2 & 3 & 4 & High \\
\hline Small & 0.700 & 2.456 & 0.241 & 0.731 & -0.333 & 19.444 & 11.308 & 6.202 & 15.261 & 14.943 \\
\hline 2 & 0.238 & 0.425 & 0.263 & -0.053 & -0.408 & 17.029 & 17.696 & 17.117 & 16.392 & 17.245 \\
\hline 3 & -1.294 & -0.377 & 1.372 & -0.856 & 0.453 & 19.507 & 19.014 & 19.696 & 19.405 & 19.013 \\
\hline 4 & 0.173 & -0.199 & 1.975 & 0.804 & 2.158 & 19.411 & 16.829 & 16.299 & 19.323 & 19.951 \\
\hline \multirow[t]{3}{*}{ Big } & 0.861 & 0.537 & 0.718 & 2.725 & 0.499 & 15.506 & 26.744 & 23.671 & 26.202 & 28.262 \\
\hline & \multicolumn{5}{|l|}{$t(s)$} & \multicolumn{5}{|l|}{$t(l)$} \\
\hline & Low & 2 & 3 & 4 & High & Low & 2 & 3 & 4 & High \\
\hline Small & 10.930 & 5.377 & 4.962 & 8.346 & 6.495 & 1.250 & 5.831 & 4.962 & 3.751 & 4.275 \\
\hline 2 & 5.335 & 6.351 & 3.503 & 6.041 & 2.400 & 5.484 & 4.979 & 3.701 & 5.285 & 4.004 \\
\hline 3 & 7.542 & 3.218 & 2.580 & 1.025 & 0.294 & 1.955 & 3.261 & 0.950 & 4.638 & 5.478 \\
\hline 4 & 2.935 & 1.593 & 0.729 & -2.495 & 1.749 & 2.191 & 4.967 & 3.519 & 7.470 & 0.046 \\
\hline Big & -1.207 & -0.394 & 0.386 & -2.813 & 0.379 & 1.389 & 0.061 & 1.427 & 3.185 & -2.429 \\
\hline
\end{tabular}

Panel E $R^{2}$

\begin{tabular}{|c|c|c|c|c|c|c|c|c|c|c|}
\hline & \multicolumn{5}{|c|}{ MC_PB } & \multicolumn{5}{|c|}{ MC_ROE } \\
\hline & Low & 2 & 3 & 4 & High & Low & 2 & 3 & 4 & High \\
\hline Small & 0.733 & 0.741 & 0.749 & 0.785 & 0.768 & 0.757 & 0.342 & 0.733 & 0.754 & 0.732 \\
\hline 2 & 0.510 & 0.716 & 0.750 & 0.793 & 0.782 & 0.755 & 0.769 & 0.751 & 0.755 & 0.760 \\
\hline 3 & 0.819 & 0.823 & 0.745 & 0.820 & 0.795 & 0.743 & 0.771 & 0.791 & 0.771 & 0.811 \\
\hline 4 & 0.854 & 0.776 & 0.812 & 0.810 & 0.768 & 0.754 & 0.782 & 0.767 & 0.747 & 0.797 \\
\hline \multirow[t]{3}{*}{ Big } & 0.876 & 0.884 & 0.908 & 0.810 & 0.847 & 0.856 & 0.868 & 0.876 & 0.839 & 0.825 \\
\hline & \multicolumn{5}{|c|}{ MC_TA } & \multicolumn{5}{|c|}{ MC_TAM } \\
\hline & Low & 2 & 3 & 4 & High & Low & 2 & 3 & 4 & High \\
\hline Small & 0.734 & 0.774 & 0.756 & 0.346 & 0.719 & 0.756 & 0.612 & 0.415 & 0.691 & 0.682 \\
\hline 2 & 0.792 & 0.734 & 0.785 & 0.750 & 0.718 & 0.740 & 0.747 & 0.722 & 0.726 & 0.730 \\
\hline 3 & 0.824 & 0.752 & 0.842 & 0.811 & 0.678 & 0.753 & 0.756 & 0.752 & 0.780 & 0.783 \\
\hline 4 & 0.721 & 0.803 & 0.798 & 0.822 & 0.737 & 0.755 & 0.735 & 0.710 & 0.813 & 0.753 \\
\hline Big & 0.795 & 0.826 & 0.900 & 0.884 & 0.847 & 0.680 & 0.852 & 0.822 & 0.864 & 0.858 \\
\hline
\end{tabular}


Table 5 Regression results of Fama-French five-factor model for 25 portfolios $R_{\mathrm{Pt}}-R_{\mathrm{Ft}}=a+b\left(R_{\mathrm{Mt}}-R_{\mathrm{Ft}}\right)+s \mathrm{SMB}_{\mathrm{t}}+l \mathrm{LMH}_{t}+p \mathrm{RMWt}+t \mathrm{CMAt}+e_{t}$

\begin{tabular}{|c|c|c|c|c|c|c|c|c|c|c|}
\hline \multicolumn{11}{|c|}{ Panel A MC_PB } \\
\hline & \multicolumn{5}{|l|}{$a$} & \multicolumn{5}{|l|}{$b$} \\
\hline & Low & 2 & 3 & 4 & High & Low & 2 & 3 & 4 & High \\
\hline Small & 0.010 & 0.011 & 0.012 & 0.003 & -0.002 & 1.063 & 1.076 & 1.100 & 0.993 & 1.087 \\
\hline 2 & 0.010 & 0.004 & 0.002 & -0.004 & 0.005 & 0.657 & 1.050 & 0.949 & 0.990 & 0.988 \\
\hline 3 & -0.004 & 0.000 & -0.001 & 0.002 & 0.003 & 1.122 & 1.176 & 0.908 & 1.104 & 0.962 \\
\hline 4 & -0.002 & 0.003 & 0.012 & 0.005 & 0.006 & 1.042 & 0.936 & 1.184 & 1.109 & 0.838 \\
\hline \multirow[t]{3}{*}{ Big } & 0.004 & 0.001 & 0.003 & 0.008 & 0.003 & 0.991 & 1.081 & 1.023 & 1.104 & 0.893 \\
\hline & \multicolumn{5}{|l|}{$S$} & \multicolumn{5}{|l|}{$L$} \\
\hline & Low & 2 & 3 & 4 & High & Low & 2 & 3 & 4 & High \\
\hline Small & 1.417 & 1.232 & 0.925 & 1.216 & 1.200 & 0.921 & 0.254 & -0.032 & -0.071 & -0.408 \\
\hline 2 & 1.697 & 0.747 & 0.761 & 0.943 & 0.659 & 0.578 & -0.001 & 0.106 & -0.018 & -0.414 \\
\hline 3 & 0.622 & 0.583 & 0.415 & 0.545 & 0.396 & 0.617 & 0.202 & 0.151 & -0.184 & -0.161 \\
\hline 4 & -0.178 & -0.097 & -0.056 & 0.304 & 0.291 & 1.042 & 0.465 & -0.350 & -0.304 & -0.387 \\
\hline \multirow[t]{3}{*}{ Big } & -0.372 & -0.371 & -0.290 & -0.157 & 0.184 & 0.922 & 0.439 & 0.278 & -0.215 & -0.484 \\
\hline & \multicolumn{5}{|l|}{$R$} & \multicolumn{5}{|l|}{$T$} \\
\hline & Low & 2 & 3 & 4 & High & Low & 2 & 3 & 4 & High \\
\hline Small & 0.384 & -0.221 & -0.241 & -0.196 & -0.288 & 0.066 & -0.086 & 0.179 & -0.132 & 0.120 \\
\hline 2 & -1.343 & -0.536 & -0.198 & -0.266 & -0.373 & -1.283 & 0.064 & 0.145 & -0.273 & -0.189 \\
\hline 3 & -0.201 & -0.359 & -0.189 & -0.228 & -0.097 & 0.260 & -0.075 & -0.023 & -0.386 & -0.195 \\
\hline 4 & -0.110 & -0.263 & -0.857 & -0.348 & -0.251 & -0.044 & -0.025 & -0.478 & -0.207 & -0.240 \\
\hline \multirow[t]{3}{*}{ Big } & -0.089 & -0.276 & -0.029 & -0.230 & -0.282 & 0.041 & -0.176 & 0.012 & -0.142 & -0.043 \\
\hline & \multicolumn{5}{|l|}{$t(a)$} & \multicolumn{5}{|l|}{$t(\mathrm{RM})$} \\
\hline & Low & 2 & 3 & 4 & High & Low & 2 & 3 & 4 & High \\
\hline Small & 1.908 & 2.216 & 2.722 & 0.875 & -0.414 & 15.406 & 16.546 & 18.995 & 20.395 & 20.935 \\
\hline 2 & 1.068 & 0.816 & 0.544 & -1.037 & 1.472 & 5.273 & 15.992 & 18.059 & 20.855 & 21.893 \\
\hline 3 & -0.935 & -0.058 & -0.341 & 0.492 & 1.076 & 19.494 & 21.491 & 17.326 & 24.189 & 22.307 \\
\hline 4 & -0.584 & 0.639 & 3.041 & 1.281 & 2.032 & 18.513 & 16.143 & 21.892 & 22.942 & 20.902 \\
\hline \multirow[t]{3}{*}{ Big } & 1.108 & 0.433 & 1.099 & 2.724 & 1.157 & 20.565 & 24.309 & 30.323 & 28.350 & 27.447 \\
\hline & \multicolumn{5}{|l|}{$t(s)$} & \multicolumn{5}{|l|}{$t(I)$} \\
\hline & Low & 2 & 3 & 4 & High & Low & 2 & 3 & 4 & High \\
\hline Small & 9.281 & 8.562 & 7.217 & 11.290 & 10.436 & 5.204 & 1.523 & -0.216 & -0.569 & -3.063 \\
\hline 2 & 6.155 & 5.143 & 6.547 & 8.973 & 6.600 & 1.807 & -0.006 & 0.786 & -0.148 & -3.580 \\
\hline 3 & 4.882 & 4.814 & 3.580 & 5.390 & 4.145 & 4.178 & 1.436 & 1.124 & -1.572 & -1.454 \\
\hline 4 & -1.425 & -0.755 & -0.467 & 2.845 & 3.280 & 7.215 & 3.124 & -2.522 & -2.456 & -3.759 \\
\hline \multirow[t]{3}{*}{ Big } & -3.483 & -3.772 & -3.889 & -1.821 & 2.555 & 7.455 & 3.851 & 3.215 & -2.151 & -5.798 \\
\hline & \multicolumn{5}{|l|}{$t(r)$} & \multicolumn{5}{|l|}{$t(T)$} \\
\hline & Low & 2 & 3 & 4 & High & Low & 2 & 3 & 4 & High \\
\hline Small & 1.958 & -1.195 & -1.466 & -1.418 & -1.949 & 0.368 & -0.509 & 1.182 & -1.042 & 0.884 \\
\hline 2 & -3.793 & -2.872 & -1.323 & -1.969 & -2.905 & -3.948 & 0.376 & 1.058 & -2.208 & -1.609 \\
\hline 3 & -1.228 & -2.306 & -1.267 & -1.757 & -0.788 & 1.735 & -0.524 & -0.168 & -3.244 & -1.737 \\
\hline 4 & -0.689 & -1.595 & -5.574 & -2.535 & -2.201 & -0.301 & -0.163 & -3.391 & -1.640 & -2.291 \\
\hline Big & -0.649 & -2.183 & -0.306 & -2.082 & -3.049 & 0.324 & -1.518 & 0.139 & -1.402 & -0.512 \\
\hline
\end{tabular}


Table 5 (continued)

\begin{tabular}{|c|c|c|c|c|c|c|c|c|c|c|}
\hline \multicolumn{11}{|c|}{ Panel B MC_ROE } \\
\hline & \multicolumn{5}{|l|}{$a$} & \multicolumn{5}{|l|}{ RM } \\
\hline & Low & 2 & 3 & 4 & High & Low & 2 & 3 & 4 & High \\
\hline Small & 0.008 & 0.019 & 0.005 & 0.004 & 0.005 & 1.088 & 0.558 & 0.995 & 1.040 & 1.126 \\
\hline 2 & -0.003 & 0.001 & 0.002 & -0.003 & 0.003 & 1.084 & 1.027 & 1.010 & 1.051 & 1.042 \\
\hline 3 & -0.001 & 0.005 & 0.000 & -0.003 & 0.007 & 1.068 & 1.062 & 1.072 & 0.975 & 1.022 \\
\hline 4 & -0.001 & 0.002 & 0.010 & 0.009 & 0.010 & 1.104 & 0.944 & 0.933 & 0.956 & 0.893 \\
\hline \multirow[t]{3}{*}{ Big } & 0.003 & 0.000 & 0.005 & 0.005 & 0.003 & 1.107 & 1.093 & 1.000 & 0.980 & 1.002 \\
\hline & \multicolumn{5}{|l|}{$S$} & \multicolumn{5}{|l|}{$L$} \\
\hline & Low & 2 & 3 & 4 & High & Low & 2 & 3 & 4 & High \\
\hline Small & 1.589 & 1.528 & 1.093 & 1.234 & 1.364 & 0.433 & -0.035 & 0.418 & 0.505 & 0.179 \\
\hline 2 & 0.796 & 0.655 & 0.611 & 0.991 & 0.659 & 0.452 & 0.204 & -0.028 & -0.061 & 0.201 \\
\hline 3 & 0.598 & 0.234 & 0.320 & 0.507 & 0.238 & 0.190 & -0.208 & 0.214 & 0.429 & 0.093 \\
\hline 4 & 0.177 & 0.023 & -0.152 & -0.111 & 0.312 & -0.132 & 0.297 & 0.111 & 0.126 & -0.274 \\
\hline \multirow[t]{3}{*}{ Big } & -0.259 & -0.229 & -0.150 & 0.034 & 0.037 & -0.007 & 0.050 & -0.075 & -0.228 & -0.079 \\
\hline & \multicolumn{5}{|l|}{$R$} & \multicolumn{5}{|l|}{$T$} \\
\hline & Low & 2 & 3 & 4 & High & Low & 2 & 3 & 4 & High \\
\hline Small & -0.165 & -2.009 & -0.051 & 0.328 & 0.365 & 0.363 & -1.874 & -0.001 & -0.254 & -0.024 \\
\hline 2 & -0.407 & -0.455 & -0.231 & -0.055 & 0.226 & 0.258 & 0.019 & 0.203 & -0.330 & -0.120 \\
\hline 3 & -0.732 & -0.863 & -0.065 & 0.127 & 0.087 & 0.019 & -0.102 & -0.133 & -0.404 & -0.193 \\
\hline 4 & -0.936 & -0.564 & -0.337 & 0.006 & -0.108 & 0.077 & -0.382 & -0.092 & 0.057 & -0.067 \\
\hline \multirow[t]{3}{*}{ Big } & -0.648 & -0.539 & -0.394 & -0.311 & 0.131 & 0.111 & -0.013 & -0.081 & -0.285 & -0.182 \\
\hline & \multicolumn{5}{|l|}{$t(a)$} & \multicolumn{5}{|l|}{$t(\mathrm{RM})$} \\
\hline & Low & 2 & 3 & 4 & High & Low & 2 & 3 & 4 & High \\
\hline Small & 1.980 & 1.718 & 1.019 & 1.025 & 1.159 & 16.267 & 3.609 & 15.935 & 18.960 & 19.054 \\
\hline 2 & -0.546 & 0.260 & 0.430 & -0.681 & 0.891 & 15.948 & 17.504 & 18.997 & 19.864 & 20.367 \\
\hline 3 & -0.284 & 1.228 & 0.120 & -0.790 & 2.175 & 15.628 & 18.865 & 20.157 & 19.533 & 23.010 \\
\hline 4 & -0.189 & 0.512 & 2.494 & 2.326 & 3.611 & 17.494 & 16.547 & 17.247 & 17.768 & 22.802 \\
\hline \multirow[t]{3}{*}{ Big } & 0.874 & -0.155 & 1.740 & 1.767 & 1.109 & 24.559 & 25.196 & 27.147 & 24.667 & 24.622 \\
\hline & \multicolumn{5}{|l|}{$t(s)$} & \multicolumn{5}{|l|}{$t(l)$} \\
\hline & Low & 2 & 3 & 4 & High & Low & 2 & 3 & 4 & High \\
\hline Small & 10.731 & 4.464 & 7.909 & 10.165 & 10.425 & 2.525 & -0.088 & 2.610 & 3.587 & 1.181 \\
\hline 2 & 5.293 & 5.045 & 5.195 & 8.465 & 5.815 & 2.590 & 1.356 & -0.206 & -0.450 & 1.528 \\
\hline 3 & 3.955 & 1.875 & 2.715 & 4.588 & 2.422 & 1.083 & -1.438 & 1.566 & 3.354 & 0.821 \\
\hline 4 & 1.271 & 0.179 & -1.269 & -0.931 & 3.594 & -0.818 & 2.028 & 0.796 & 0.912 & -2.729 \\
\hline \multirow[t]{3}{*}{ Big } & -2.591 & -2.385 & -1.836 & 0.384 & 0.407 & -0.061 & 0.447 & -0.795 & -2.238 & -0.760 \\
\hline & \multicolumn{5}{|l|}{$t(r)$} & \multicolumn{5}{|l|}{$t(T)$} \\
\hline & Low & 2 & 3 & 4 & High & Low & 2 & 3 & 4 & High \\
\hline Small & -0.868 & -4.569 & -0.285 & 2.106 & 2.173 & 2.082 & -4.645 & -0.007 & -1.772 & -0.154 \\
\hline 2 & -2.106 & -2.727 & -1.527 & -0.364 & 1.550 & 1.456 & 0.124 & 1.467 & -2.393 & -0.899 \\
\hline 3 & -3.767 & -5.395 & -0.430 & 0.893 & 0.691 & 0.108 & -0.692 & -0.960 & -3.100 & -1.668 \\
\hline 4 & -5.220 & -3.480 & -2.191 & 0.036 & -0.970 & 0.469 & -2.569 & -0.650 & 0.409 & -0.655 \\
\hline Big & -5.055 & -4.372 & -3.756 & -2.752 & 1.133 & 0.942 & -0.117 & -0.848 & -2.751 & -1.716 \\
\hline
\end{tabular}


Table 5 (continued)

\begin{tabular}{|c|c|c|c|c|c|c|c|c|c|c|}
\hline \multicolumn{11}{|c|}{ Panel C MC_TA } \\
\hline & \multicolumn{5}{|l|}{$a$} & \multicolumn{5}{|l|}{$\mathrm{RM}$} \\
\hline & Low & 2 & 3 & 4 & High & Low & 2 & 3 & 4 & High \\
\hline Small & 0.010 & 0.006 & 0.002 & 0.018 & 0.005 & 1.098 & 1.156 & 1.074 & 0.592 & 1.089 \\
\hline 2 & 0.001 & 0.002 & 0.001 & 0.002 & 0.001 & 1.082 & 1.038 & 0.950 & 0.917 & 1.022 \\
\hline 3 & -0.001 & -0.002 & -0.003 & 0.001 & -0.003 & 1.110 & 0.996 & 1.124 & 1.097 & 1.012 \\
\hline 4 & 0.004 & 0.008 & 0.007 & 0.003 & 0.011 & 1.036 & 1.002 & 0.986 & 0.963 & 1.189 \\
\hline \multirow[t]{3}{*}{ Big } & 0.007 & 0.004 & 0.001 & 0.008 & 0.003 & 0.968 & 0.829 & 0.996 & 1.020 & 1.109 \\
\hline & \multicolumn{5}{|l|}{$S$} & \multicolumn{5}{|l|}{$L$} \\
\hline & Low & 2 & 3 & 4 & High & Low & 2 & 3 & 4 & High \\
\hline Small & 1.438 & 1.246 & 1.055 & 1.638 & 1.528 & 0.214 & 0.402 & 0.415 & 0.028 & 0.479 \\
\hline 2 & 0.654 & 0.662 & 0.652 & 0.449 & 0.933 & 0.383 & 0.034 & 0.150 & 0.253 & 0.070 \\
\hline 3 & 0.553 & 0.297 & 0.378 & 0.201 & 0.712 & 0.188 & 0.194 & 0.166 & 0.075 & 0.235 \\
\hline 4 & 0.143 & -0.022 & 0.003 & 0.031 & 0.120 & -0.137 & 0.014 & 0.205 & 0.238 & -0.532 \\
\hline \multirow[t]{3}{*}{ Big } & -0.029 & -0.164 & -0.122 & -0.254 & 0.066 & -0.245 & 0.128 & -0.037 & 0.232 & -0.422 \\
\hline & \multicolumn{5}{|l|}{$R$} & \multicolumn{5}{|l|}{$T$} \\
\hline & Low & 2 & 3 & 4 & High & Low & 2 & 3 & 4 & High \\
\hline Small & -0.187 & -0.109 & -0.038 & -1.517 & 0.065 & 0.716 & 0.322 & 0.083 & -1.940 & -0.383 \\
\hline 2 & -0.182 & -0.366 & -0.376 & -0.196 & -0.396 & 0.207 & 0.169 & 0.031 & -0.020 & -0.724 \\
\hline 3 & -0.283 & -0.113 & -0.106 & -0.199 & -0.304 & 0.460 & 0.416 & -0.155 & -0.199 & -1.101 \\
\hline 4 & -0.399 & -0.366 & -0.145 & -0.170 & -0.925 & 0.134 & 0.096 & 0.098 & -0.214 & -0.742 \\
\hline \multirow[t]{3}{*}{ Big } & -0.419 & -0.169 & -0.139 & 0.057 & -0.681 & 0.350 & 0.142 & -0.003 & -0.238 & -0.603 \\
\hline & \multicolumn{5}{|l|}{$t(a)$} & \multicolumn{5}{|l|}{$t(\mathrm{RM})$} \\
\hline & Low & 2 & 3 & 4 & High & Low & 2 & 3 & 4 & High \\
\hline Small & 2.316 & 1.385 & 0.373 & 1.803 & 1.014 & 18.752 & 18.242 & 17.314 & 4.406 & 15.812 \\
\hline 2 & 0.350 & 0.441 & 0.146 & 0.407 & 0.329 & 19.149 & 17.292 & 18.852 & 16.965 & 16.805 \\
\hline 3 & -0.236 & -0.569 & -1.024 & 0.250 & -0.727 & 23.439 & 18.659 & 24.216 & 21.480 & 15.793 \\
\hline 4 & 0.836 & 2.131 & 2.035 & 0.935 & 2.314 & 17.092 & 20.515 & 19.855 & 20.993 & 18.111 \\
\hline \multirow[t]{3}{*}{ Big } & 2.110 & 1.291 & 0.342 & 3.054 & 1.014 & 22.806 & 21.628 & 31.404 & 28.133 & 27.144 \\
\hline & \multicolumn{5}{|l|}{$t(s)$} & \multicolumn{5}{|l|}{$t(I)$} \\
\hline & Low & 2 & 3 & 4 & High & Low & 2 & 3 & 4 & High \\
\hline Small & 11.095 & 8.881 & 7.684 & 5.504 & 10.026 & 1.428 & 2.471 & 2.610 & 0.082 & 2.710 \\
\hline 2 & 5.227 & 4.978 & 5.846 & 3.753 & 6.928 & 2.641 & 0.221 & 1.158 & 1.822 & 0.446 \\
\hline 3 & 5.271 & 2.516 & 3.683 & 1.775 & 5.022 & 1.548 & 1.416 & 1.397 & 0.570 & 1.429 \\
\hline 4 & 1.067 & -0.207 & 0.031 & 0.308 & 0.826 & -0.879 & 0.111 & 1.609 & 2.025 & -3.161 \\
\hline \multirow[t]{3}{*}{ Big } & -0.307 & -1.937 & -1.740 & -3.167 & 0.734 & -2.253 & 1.307 & -0.461 & 2.493 & -4.027 \\
\hline & \multicolumn{5}{|l|}{$t(r)$} & \multicolumn{5}{|l|}{$t(T)$} \\
\hline & Low & 2 & 3 & 4 & High & Low & 2 & 3 & 4 & High \\
\hline Small & -1.126 & -0.605 & -0.218 & -3.970 & 0.330 & 4.690 & 1.950 & 0.516 & -5.531 & -2.134 \\
\hline 2 & -1.130 & -2.142 & -2.623 & -1.275 & -2.292 & 1.401 & 1.078 & 0.235 & -0.143 & -4.562 \\
\hline 3 & -2.100 & -0.744 & -0.804 & -1.371 & -1.667 & 3.724 & 2.986 & -1.279 & -1.493 & -6.585 \\
\hline 4 & -2.315 & -2.638 & -1.028 & -1.306 & -4.957 & 0.849 & 0.750 & 0.754 & -1.788 & -4.330 \\
\hline Big & -3.471 & -1.548 & -1.539 & 0.554 & -5.859 & 3.158 & 1.423 & -0.039 & -2.519 & -5.657 \\
\hline
\end{tabular}


Table 5 (continued)

\begin{tabular}{|c|c|c|c|c|c|c|c|c|c|c|}
\hline \multicolumn{11}{|c|}{ Panel D MC_TAM } \\
\hline & \multicolumn{5}{|l|}{$a$} & \multicolumn{5}{|l|}{ RM } \\
\hline & Low & 2 & 3 & 4 & High & Low & 2 & 3 & 4 & High \\
\hline Small & 0.003 & 0.014 & 0.009 & 0.004 & -0.004 & 1.317 & 0.845 & 0.673 & 0.962 & 0.934 \\
\hline 2 & 0.005 & 0.006 & 0.002 & -0.001 & -0.001 & 1.170 & 0.989 & 0.982 & 0.938 & 0.935 \\
\hline 3 & -0.003 & 0.001 & 0.008 & -0.002 & 0.002 & 1.212 & 1.037 & 1.031 & 1.085 & 0.901 \\
\hline 4 & 0.005 & 0.000 & 0.007 & 0.004 & 0.007 & 1.158 & 0.837 & 0.867 & 0.891 & 0.923 \\
\hline \multirow[t]{3}{*}{ Big } & 0.009 & 0.005 & 0.003 & 0.008 & 0.003 & 0.947 & 1.264 & 1.069 & 0.956 & 0.985 \\
\hline & \multicolumn{5}{|l|}{$S$} & \multicolumn{5}{|l|}{$L$} \\
\hline & Low & 2 & 3 & 4 & High & Low & 2 & 3 & 4 & High \\
\hline Small & 1.544 & 0.900 & 1.717 & 1.135 & 0.817 & 0.152 & 0.581 & 0.277 & 0.294 & 0.643 \\
\hline 2 & 0.814 & 0.798 & 0.399 & 0.741 & 0.269 & 0.234 & 0.047 & 0.238 & 0.570 & 0.264 \\
\hline 3 & 1.031 & 0.348 & 0.298 & 0.221 & 0.021 & -0.070 & 0.035 & -0.141 & 0.286 & 0.324 \\
\hline 4 & 0.388 & 0.149 & 0.168 & -0.245 & 0.160 & -0.224 & 0.296 & 0.143 & 0.392 & 0.035 \\
\hline \multirow[t]{3}{*}{ Big } & -0.166 & 0.018 & 0.060 & -0.231 & 0.028 & -0.345 & -0.281 & 0.009 & 0.064 & -0.325 \\
\hline & \multicolumn{5}{|l|}{$R$} & \multicolumn{5}{|l|}{$T$} \\
\hline & Low & 2 & 3 & 4 & High & Low & 2 & 3 & 4 & High \\
\hline Small & 0.104 & -0.105 & -1.180 & -0.051 & 0.383 & 0.357 & -0.005 & -1.397 & 0.140 & 0.285 \\
\hline 2 & -0.435 & -0.490 & -0.062 & 0.174 & -0.063 & 0.033 & 0.009 & 0.207 & 0.096 & 0.091 \\
\hline 3 & -0.312 & -0.264 & -0.278 & -0.241 & -0.081 & 0.074 & 0.183 & 0.002 & -0.458 & 0.039 \\
\hline 4 & -0.546 & -0.072 & -0.273 & -0.193 & 0.060 & 0.000 & 0.124 & -0.410 & -0.095 & 0.098 \\
\hline \multirow[t]{3}{*}{ Big } & -0.630 & -0.432 & -0.137 & -0.147 & -0.254 & -0.092 & -0.342 & -0.116 & -0.011 & -0.017 \\
\hline & \multicolumn{5}{|l|}{$t(a)$} & \multicolumn{5}{|l|}{$t(\mathrm{RM})$} \\
\hline & Low & 2 & 3 & 4 & High & Low & 2 & 3 & 4 & High \\
\hline Small & 0.637 & 2.478 & 0.981 & 0.837 & -0.896 & 19.324 & 10.802 & 5.219 & 14.804 & 15.251 \\
\hline 2 & 0.914 & 1.357 & 0.457 & -0.338 & -0.223 & 16.360 & 17.119 & 16.745 & 16.075 & 16.655 \\
\hline 3 & -0.680 & 0.241 & 1.880 & -0.579 & 0.632 & 18.807 & 18.607 & 18.885 & 18.519 & 18.273 \\
\hline 4 & 1.151 & 0.026 & 1.731 & 1.158 & 1.964 & 18.841 & 16.329 & 15.398 & 18.368 & 19.411 \\
\hline \multirow[t]{3}{*}{ Big } & 1.907 & 1.329 & 0.943 & 3.020 & 1.250 & 14.882 & 25.835 & 22.560 & 25.124 & 27.341 \\
\hline & \multicolumn{5}{|l|}{$t(s)$} & \multicolumn{5}{|l|}{$t(l)$} \\
\hline & Low & 2 & 3 & 4 & High & Low & 2 & 3 & 4 & High \\
\hline Small & 10.232 & 5.194 & 6.015 & 7.886 & 6.028 & 0.871 & 2.895 & 0.837 & 1.762 & 4.093 \\
\hline 2 & 5.146 & 6.241 & 3.078 & 5.738 & 2.164 & 1.274 & 0.317 & 1.580 & 3.807 & 1.832 \\
\hline 3 & 7.232 & 2.826 & 2.469 & 1.702 & 0.195 & -0.423 & 0.247 & -1.007 & 1.906 & 2.558 \\
\hline 4 & 2.850 & 1.310 & 1.349 & -2.286 & 1.516 & -1.422 & 2.252 & 0.992 & 3.153 & 0.291 \\
\hline \multirow[t]{3}{*}{ Big } & -1.177 & 0.163 & 0.572 & -2.747 & 0.349 & -2.112 & -2.241 & 0.071 & 0.657 & -3.519 \\
\hline & \multicolumn{5}{|l|}{$t(r)$} & \multicolumn{5}{|l|}{$t(T)$} \\
\hline & Low & 2 & 3 & 4 & High & Low & 2 & 3 & 4 & High \\
\hline Small & 0.535 & -0.470 & -3.218 & -0.275 & 2.201 & 2.008 & -0.027 & -4.151 & 0.825 & 1.785 \\
\hline 2 & -2.142 & -2.981 & -0.372 & 1.047 & -0.394 & 0.175 & 0.059 & 1.354 & 0.628 & 0.621 \\
\hline 3 & -1.704 & -1.667 & -1.789 & -1.446 & -0.575 & 0.440 & 1.262 & 0.016 & -2.999 & 0.306 \\
\hline 4 & -3.125 & -0.494 & -1.708 & -1.399 & 0.446 & -0.002 & 0.928 & -2.794 & -0.749 & 0.794 \\
\hline Big & -3.483 & -3.105 & -1.021 & -1.355 & -2.478 & -0.557 & -2.680 & -0.940 & -0.115 & -0.184 \\
\hline
\end{tabular}


Table 5 (continued)

\begin{tabular}{|c|c|c|c|c|c|c|c|c|c|c|}
\hline \multicolumn{11}{|c|}{ Panel E $R^{2}$} \\
\hline & \multicolumn{5}{|c|}{ MC_PB } & \multicolumn{5}{|c|}{ MC_ROE } \\
\hline & Low & 2 & 3 & 4 & High & Low & 2 & 3 & 4 & High \\
\hline Small & 0.740 & 0.744 & 0.762 & 0.789 & 0.777 & 0.768 & 0.452 & 0.733 & 0.771 & 0.741 \\
\hline 2 & 0.571 & 0.734 & 0.757 & 0.801 & 0.794 & 0.770 & 0.782 & 0.762 & 0.764 & 0.768 \\
\hline 3 & 0.827 & 0.829 & 0.747 & 0.832 & 0.799 & 0.768 & 0.810 & 0.792 & 0.790 & 0.817 \\
\hline 4 & 0.854 & 0.780 & 0.845 & 0.818 & 0.778 & 0.798 & 0.800 & 0.774 & 0.747 & 0.799 \\
\hline \multirow[t]{3}{*}{ Big } & 0.876 & 0.888 & 0.908 & 0.881 & 0.856 & 0.882 & 0.884 & 0.886 & 0.850 & 0.832 \\
\hline & \multicolumn{5}{|c|}{ MC_TA } & \multicolumn{5}{|c|}{ MC_TAM } \\
\hline & Low & 2 & 3 & 4 & High & Low & 2 & 3 & 4 & High \\
\hline Small & 0.800 & 0.782 & 0.757 & 0.467 & 0.730 & 0.763 & 0.613 & 0.484 & 0.693 & 0.694 \\
\hline 2 & 0.799 & 0.748 & 0.797 & 0.753 & 0.753 & 0.749 & 0.763 & 0.727 & 0.728 & 0.732 \\
\hline 3 & 0.851 & 0.771 & 0.845 & 0.815 & 0.750 & 0.759 & 0.766 & 0.757 & 0.792 & 0.784 \\
\hline 4 & 0.736 & 0.816 & 0.802 & 0.827 & 0.782 & 0.771 & 0.738 & 0.725 & 0.815 & 0.754 \\
\hline Big & 0.833 & 0.834 & 0.902 & 0.890 & 0.884 & 0.705 & 0.863 & 0.824 & 0.865 & 0.863 \\
\hline
\end{tabular}

for microcap portfolio indicate that first portfolio with low profitability microcaps is dominated by firm whose stocks behave like unprofitable firms with aggressive investment.

MC_TA: Six portfolios found to be significant with average alpha value of 0.0043 and 78.9 average $R$-square (\%). Here also like previous case high positive investment (CMA) and less negative profitability (RMW) coefficient for microcap portfolio indicate that first portfolio with low profitability microcaps is dominated by firm whose stocks behave like unprofitable firms with aggressive investment and the problem with microcap remains unsolved.

MC_TAM: Four portfolios found to be significant with average alpha value of 0.0047 and 74.9 average $R$-square (\%). The biggest problem lies with Fama-French (2015) is microcaps due to high negative profitability (RMW) and investment (CMA) factor but it is well captured with MC_TAM cross portfolio. So, it indicates that it is not the asset pricing problem but the way how portfolios constructed are of much importance.

From the above discussion, it is very much clear that microcap is the problem for most of the crosses as similar to Fama-French (2015) findings. Study result also confirms that Fama-French (2015) five model is not better than Fama-French (1993) three-factor model in Indian context. Further study confirms Fama-French five-factor model is not a global similar to studies by Hou et al. (2014), Clarke (2016), and Chiah et al. (2016).

Then study runs modified five-factor model with human asset investment with market $(\mathrm{Rm})$, size (SMB), value (LMH), profitability (RMW), and investment with human asset (CvMAv).

\subsection{Modified five-factor regressions (with human asset investment)}

The regressions' result of the modified five-factor model with human asset investment is shown in Table 6. 
Table 6 Regression results of Modified Five-Factor Model with human asset for 25 portfolios $R_{\mathrm{Pt}}-R_{\mathrm{Ft}}=a+b\left(R_{\mathrm{Mt}}-R_{\mathrm{Ft}}\right)+s \mathrm{SMB}_{\mathrm{t}}+I \mathrm{LMH}_{t}+p \mathrm{RMWt}+h \mathrm{CvMAvt}+e_{t}$

\begin{tabular}{|c|c|c|c|c|c|c|c|c|c|c|}
\hline \multicolumn{11}{|c|}{ Panel A MC_PB } \\
\hline & \multicolumn{5}{|l|}{$a$} & \multicolumn{5}{|l|}{$b$} \\
\hline & Low & 2 & 3 & 4 & High & Low & 2 & 3 & 4 & High \\
\hline Small & 0.007 & 0.009 & 0.011 & 0.002 & -0.001 & 0.950 & 1.017 & 1.034 & 0.971 & 1.093 \\
\hline 2 & 0.010 & 0.003 & 0.001 & -0.004 & 0.004 & 0.833 & 0.987 & 0.886 & 1.017 & 0.977 \\
\hline 3 & -0.004 & -0.001 & -0.001 & 0.001 & 0.003 & 1.099 & 1.145 & 0.930 & 1.123 & 0.957 \\
\hline 4 & -0.003 & 0.002 & 0.010 & 0.003 & 0.005 & 1.021 & 0.896 & 1.145 & 1.062 & 0.814 \\
\hline \multirow[t]{3}{*}{ Big } & 0.004 & 0.001 & 0.003 & 0.007 & 0.002 & 0.984 & 1.097 & 1.014 & 1.100 & 0.883 \\
\hline & \multicolumn{5}{|l|}{$S$} & \multicolumn{5}{|l|}{$L$} \\
\hline & Low & 2 & 3 & 4 & High & Low & 2 & 3 & 4 & High \\
\hline Small & 1.157 & 1.047 & 0.828 & 1.109 & 1.262 & 1.128 & 0.402 & 0.045 & 0.014 & -0.458 \\
\hline 2 & 1.634 & 0.613 & 0.660 & 0.902 & 0.557 & 0.629 & 0.106 & 0.186 & 0.015 & -0.333 \\
\hline 3 & 0.666 & 0.475 & 0.463 & 0.439 & 0.304 & 0.582 & 0.287 & 0.114 & -0.100 & -0.088 \\
\hline 4 & -0.248 & -0.208 & -0.345 & 0.103 & 0.133 & 1.098 & 0.553 & -0.120 & -0.144 & -0.261 \\
\hline \multirow[t]{3}{*}{ Big } & -0.374 & -0.400 & -0.308 & -0.223 & 0.140 & 0.924 & 0.462 & 0.293 & -0.162 & -0.449 \\
\hline & \multicolumn{5}{|l|}{$R$} & \multicolumn{5}{|l|}{$\mathrm{h}$} \\
\hline & Low & 2 & 3 & 4 & High & Low & 2 & 3 & 4 & High \\
\hline Small & 0.641 & -0.012 & -0.175 & -0.060 & -0.375 & 0.665 & 0.410 & 0.320 & 0.197 & -0.095 \\
\hline 2 & -1.033 & -0.409 & -0.120 & -0.171 & -0.231 & -0.458 & 0.358 & 0.315 & -0.031 & 0.158 \\
\hline 3 & -0.296 & -0.233 & -0.233 & -0.045 & 0.036 & 0.017 & 0.227 & -0.126 & 0.073 & 0.130 \\
\hline 4 & -0.029 & -0.143 & -0.466 & -0.100 & -0.041 & 0.151 & 0.258 & 0.475 & 0.391 & 0.271 \\
\hline \multirow[t]{3}{*}{ Big } & -0.095 & -0.213 & -0.013 & -0.134 & -0.228 & 0.025 & -0.014 & 0.049 & 0.094 & 0.087 \\
\hline & \multicolumn{5}{|l|}{$t(a)$} & \multicolumn{5}{|l|}{$t(\mathrm{RM})$} \\
\hline & Low & 2 & 3 & 4 & High & Low & 2 & 3 & 4 & High \\
\hline Small & 1.543 & 1.924 & 2.535 & 0.635 & -0.284 & 13.802 & 15.171 & 17.172 & 19.059 & 19.974 \\
\hline 2 & 1.006 & 0.582 & 0.314 & -1.089 & 1.221 & 6.094 & 14.510 & 16.288 & 20.033 & 20.538 \\
\hline 3 & -0.854 & -0.280 & -0.235 & 0.251 & 0.842 & 17.952 & 20.050 & 16.908 & 22.639 & 20.965 \\
\hline 4 & -0.724 & 0.423 & 2.464 & 0.839 & 1.608 & 17.293 & 14.842 & 20.224 & 21.469 & 19.427 \\
\hline \multirow[t]{3}{*}{ Big } & 1.093 & 0.371 & 1.032 & 2.522 & 1.004 & 19.383 & 23.261 & 28.576 & 26.745 & 25.836 \\
\hline & \multicolumn{5}{|l|}{$t(s)$} & \multicolumn{5}{|l|}{$t(I)$} \\
\hline & Low & 2 & 3 & 4 & High & Low & 2 & 3 & 4 & High \\
\hline Small & 7.459 & 6.935 & 6.107 & 9.664 & 10.241 & 6.505 & 2.380 & 0.294 & 0.109 & -3.322 \\
\hline 2 & 5.309 & 3.999 & 5.385 & 7.892 & 5.195 & 1.826 & 0.618 & 1.359 & 0.114 & -2.777 \\
\hline 3 & 4.832 & 3.693 & 3.734 & 3.928 & 2.958 & 3.774 & 1.997 & 0.819 & -0.797 & -0.764 \\
\hline 4 & -1.867 & -1.529 & -2.705 & 0.924 & 1.408 & 7.382 & 3.635 & -0.840 & -1.157 & -2.471 \\
\hline \multirow[t]{3}{*}{ Big } & -3.268 & -3.766 & -3.857 & -2.411 & 1.815 & 7.221 & 3.891 & 3.272 & -1.563 & -5.214 \\
\hline & \multicolumn{5}{|l|}{$t(r)$} & \multicolumn{5}{|l|}{$t(h)$} \\
\hline & Low & 2 & 3 & 4 & High & Low & 2 & 3 & 4 & High \\
\hline Small & 3.450 & -0.067 & -1.079 & -0.438 & -2.540 & 4.217 & 2.670 & 2.320 & 1.684 & -0.759 \\
\hline 2 & -2.800 & -2.227 & -0.820 & -1.250 & -1.795 & -1.461 & 2.295 & 2.529 & -0.269 & 1.453 \\
\hline 3 & -1.792 & -1.509 & -1.571 & -0.334 & 0.289 & 0.122 & 1.731 & -1.000 & 0.645 & 1.240 \\
\hline 4 & -0.179 & -0.878 & -3.051 & -0.751 & -0.365 & 1.115 & 1.865 & 3.657 & 3.449 & 2.821 \\
\hline Big & -0.690 & -1.669 & -0.137 & -1.211 & -2.470 & 0.211 & -0.129 & 0.607 & 0.994 & 1.114 \\
\hline
\end{tabular}


Table 6 (continued)

\begin{tabular}{|c|c|c|c|c|c|c|c|c|c|c|}
\hline \multicolumn{11}{|c|}{ Panel B MC_ROE } \\
\hline & \multicolumn{5}{|l|}{$a$} & \multicolumn{5}{|l|}{$b$} \\
\hline & Low & 2 & 3 & 4 & High & Low & 2 & 3 & 4 & High \\
\hline Small & 0.007 & 0.017 & 0.004 & 0.003 & 0.004 & 1.013 & 0.727 & 0.964 & 1.030 & 1.082 \\
\hline 2 & -0.003 & 0.000 & 0.002 & -0.003 & 0.003 & 1.041 & 0.983 & 0.982 & 1.095 & 1.065 \\
\hline 3 & -0.003 & 0.004 & 0.000 & -0.003 & 0.005 & 0.979 & 1.043 & 1.054 & 1.016 & 0.966 \\
\hline 4 & -0.002 & 0.001 & 0.009 & 0.008 & 0.010 & 1.049 & 0.938 & 0.917 & 0.915 & 0.873 \\
\hline \multirow[t]{3}{*}{ Big } & 0.002 & -0.001 & 0.005 & 0.004 & 0.003 & 1.073 & 1.076 & 1.018 & 0.983 & 0.996 \\
\hline & \multicolumn{5}{|l|}{$S$} & \multicolumn{5}{|l|}{$L$} \\
\hline & Low & 2 & 3 & 4 & High & Low & 2 & 3 & 4 & High \\
\hline Small & 1.542 & 1.212 & 1.014 & 1.108 & 1.241 & 0.471 & 0.218 & 0.481 & 0.606 & 0.277 \\
\hline 2 & 0.791 & 0.549 & 0.622 & 0.972 & 0.668 & 0.455 & 0.289 & -0.036 & -0.046 & 0.193 \\
\hline 3 & 0.379 & 0.145 & 0.222 & 0.451 & 0.020 & 0.364 & -0.137 & 0.292 & 0.474 & 0.267 \\
\hline 4 & 0.068 & -0.143 & -0.228 & -0.194 & 0.234 & -0.045 & 0.429 & 0.171 & 0.192 & -0.213 \\
\hline \multirow[t]{3}{*}{ Big } & -0.301 & -0.277 & -0.138 & -0.071 & -0.051 & 0.027 & 0.088 & -0.084 & -0.144 & -0.010 \\
\hline & \multicolumn{5}{|l|}{$R$} & \multicolumn{5}{|l|}{$h$} \\
\hline & Low & 2 & 3 & 4 & High & Low & 2 & 3 & 4 & High \\
\hline Small & -0.185 & -1.324 & 0.032 & 0.508 & 0.497 & 0.288 & -0.124 & 0.193 & 0.187 & 0.288 \\
\hline 2 & -0.451 & -0.349 & -0.280 & 0.028 & 0.238 & 0.135 & 0.268 & 0.072 & -0.112 & -0.081 \\
\hline 3 & -0.509 & -0.752 & 0.062 & 0.261 & 0.350 & 0.541 & 0.168 & 0.175 & -0.057 & 0.438 \\
\hline 4 & -0.837 & -0.320 & -0.240 & 0.080 & -0.015 & 0.304 & 0.221 & 0.142 & 0.228 & 0.156 \\
\hline \multirow[t]{3}{*}{ Big } & -0.625 & -0.487 & -0.390 & -0.148 & 0.256 & 0.156 & 0.110 & -0.067 & 0.119 & 0.125 \\
\hline & \multicolumn{5}{|l|}{$t(a)$} & \multicolumn{5}{|l|}{$t(b)$} \\
\hline & Low & 2 & 3 & 4 & High & Low & 2 & 3 & 4 & High \\
\hline Small & 1.502 & 1.430 & 0.872 & 0.772 & 0.928 & 14.323 & 4.186 & 14.738 & 17.767 & 17.609 \\
\hline 2 & -0.565 & 0.050 & 0.431 & -0.681 & 0.917 & 14.486 & 16.084 & 17.441 & 19.352 & 19.736 \\
\hline 3 & -0.674 & 1.052 & -0.081 & -0.853 & 1.698 & 14.103 & 17.653 & 18.884 & 18.775 & 21.662 \\
\hline 4 & -0.397 & 0.203 & 2.332 & 2.153 & 3.398 & 15.977 & 15.427 & 16.143 & 16.296 & 21.330 \\
\hline \multirow[t]{3}{*}{ Big } & 0.754 & -0.280 & 1.776 & 1.458 & 0.873 & 22.691 & 23.645 & 26.214 & 23.062 & 23.150 \\
\hline & \multicolumn{5}{|l|}{$t(s)$} & \multicolumn{5}{|l|}{$t(l)$} \\
\hline & Low & 2 & 3 & 4 & High & Low & 2 & 3 & 4 & High \\
\hline Small & 9.679 & 3.097 & 6.881 & 8.484 & 8.969 & 2.643 & 0.498 & 2.921 & 4.146 & 1.789 \\
\hline 2 & 4.886 & 3.990 & 4.901 & 7.630 & 5.501 & 2.514 & 1.876 & -0.257 & -0.322 & 1.419 \\
\hline 3 & 2.426 & 1.087 & 1.762 & 3.702 & 0.199 & 2.080 & -0.919 & 2.074 & 3.477 & 2.374 \\
\hline 4 & 0.458 & -1.044 & -1.785 & -1.531 & 2.539 & -0.273 & 2.797 & 1.197 & 1.355 & -2.061 \\
\hline \multirow[t]{3}{*}{ Big } & -2.826 & -2.700 & -1.579 & -0.741 & -0.521 & 0.223 & 0.764 & -0.862 & -1.345 & -0.091 \\
\hline & \multicolumn{5}{|l|}{$t(r)$} & \multicolumn{5}{|l|}{$t(h)$} \\
\hline & Low & 2 & 3 & 4 & High & Low & 2 & 3 & 4 & High \\
\hline Small & -0.971 & -2.821 & 0.181 & 3.243 & 2.997 & 1.779 & -0.311 & 1.285 & 1.404 & 2.044 \\
\hline 2 & -2.323 & -2.113 & -1.844 & 0.181 & 1.636 & 0.820 & 1.912 & 0.555 & -0.861 & -0.654 \\
\hline 3 & -2.715 & -4.714 & 0.409 & 1.788 & 2.903 & 3.399 & 1.241 & 1.365 & -0.459 & 4.281 \\
\hline 4 & -4.726 & -1.948 & -1.566 & 0.529 & -0.137 & 2.018 & 1.582 & 1.092 & 1.774 & 1.666 \\
\hline Big & -4.895 & -3.964 & -3.721 & -1.284 & 2.204 & 1.439 & 1.052 & -0.755 & 1.220 & 1.270 \\
\hline
\end{tabular}


Table 6 (continued)

\begin{tabular}{|c|c|c|c|c|c|c|c|c|c|c|}
\hline \multicolumn{11}{|c|}{ Panel C MC_TA } \\
\hline & \multicolumn{5}{|l|}{$a$} & \multicolumn{5}{|l|}{$b$} \\
\hline & Low & 2 & 3 & 4 & High & Low & 2 & 3 & 4 & High \\
\hline Small & 0.009 & 0.005 & 0.001 & 0.017 & 0.003 & 0.968 & 1.034 & 1.007 & 0.829 & 1.056 \\
\hline 2 & 0.001 & 0.001 & 0.000 & 0.001 & 0.001 & 1.025 & 0.991 & 0.940 & 0.912 & 1.087 \\
\hline 3 & -0.001 & -0.002 & -0.004 & 0.000 & -0.005 & 1.039 & 0.935 & 1.126 & 1.064 & 1.096 \\
\hline 4 & 0.003 & 0.007 & 0.007 & 0.003 & 0.008 & 0.986 & 0.951 & 0.955 & 0.978 & 1.148 \\
\hline \multirow[t]{3}{*}{ Big } & 0.007 & 0.005 & 0.001 & 0.007 & 0.001 & 0.934 & 0.856 & 0.991 & 1.005 & 1.105 \\
\hline & \multicolumn{5}{|l|}{$S$} & \multicolumn{5}{|l|}{$L$} \\
\hline & Low & 2 & 3 & 4 & High & Low & 2 & 3 & 4 & High \\
\hline Small & 1.393 & 1.063 & 0.918 & 1.467 & 1.292 & 0.249 & 0.547 & 0.524 & 0.166 & 0.667 \\
\hline 2 & 0.590 & 0.608 & 0.639 & 0.428 & 0.809 & 0.434 & 0.077 & 0.160 & 0.269 & 0.169 \\
\hline 3 & 0.555 & 0.306 & 0.322 & 0.039 & 0.487 & 0.186 & 0.187 & 0.212 & 0.203 & 0.415 \\
\hline 4 & 0.069 & -0.115 & -0.037 & -0.015 & -0.280 & -0.078 & 0.087 & 0.237 & 0.275 & -0.214 \\
\hline \multirow[t]{3}{*}{ Big } & 0.023 & -0.038 & -0.135 & -0.387 & -0.185 & -0.287 & 0.028 & -0.027 & 0.338 & -0.222 \\
\hline & \multicolumn{5}{|l|}{$R$} & \multicolumn{5}{|l|}{$h$} \\
\hline & Low & 2 & 3 & 4 & High & Low & 2 & 3 & 4 & High \\
\hline Small & -0.278 & 0.019 & 0.087 & -0.970 & 0.383 & 0.450 & 0.598 & 0.372 & -0.508 & 0.393 \\
\hline 2 & -0.155 & -0.342 & -0.368 & -0.171 & -0.130 & 0.254 & 0.211 & 0.047 & 0.041 & -0.043 \\
\hline 3 & -0.373 & -0.201 & -0.018 & 0.006 & 0.139 & 0.213 & 0.177 & 0.065 & 0.298 & 0.023 \\
\hline 4 & -0.348 & -0.289 & -0.122 & -0.082 & -0.369 & 0.243 & 0.270 & 0.144 & 0.010 & 0.620 \\
\hline \multirow[t]{3}{*}{ Big } & -0.540 & -0.327 & -0.124 & 0.240 & -0.305 & 0.040 & -0.240 & 0.031 & 0.210 & 0.324 \\
\hline & \multicolumn{5}{|l|}{$t(a)$} & \multicolumn{5}{|l|}{$t(b)$} \\
\hline & Low & 2 & 3 & 4 & High & Low & 2 & 3 & 4 & High \\
\hline Small & 2.086 & 1.066 & 0.116 & 1.572 & 0.641 & 15.137 & 16.100 & 15.723 & 5.385 & 14.610 \\
\hline 2 & 0.204 & 0.325 & 0.112 & 0.363 & 0.135 & 17.303 & 15.719 & 17.718 & 16.027 & 15.933 \\
\hline 3 & -0.262 & -0.568 & -1.140 & -0.098 & -0.930 & 20.169 & 16.257 & 22.939 & 20.032 & 14.357 \\
\hline 4 & 0.688 & 1.925 & 1.928 & 0.827 & 1.633 & 15.551 & 18.772 & 18.313 & 20.050 & 16.423 \\
\hline \multirow[t]{3}{*}{ Big } & 2.138 & 1.672 & 0.293 & 2.641 & 0.344 & 20.259 & 21.547 & 29.695 & 26.274 & 24.082 \\
\hline & \multicolumn{5}{|l|}{$t(s)$} & \multicolumn{5}{|l|}{$t(l)$} \\
\hline & Low & 2 & 3 & 4 & High & Low & 2 & 3 & 4 & High \\
\hline Small & 9.668 & 7.349 & 6.364 & 4.232 & 7.934 & 1.547 & 3.380 & 3.247 & 0.428 & 3.664 \\
\hline 2 & 4.421 & 4.282 & 5.345 & 3.341 & 5.264 & 2.907 & 0.482 & 1.199 & 1.878 & 0.984 \\
\hline 3 & 4.782 & 2.362 & 2.908 & 0.327 & 2.834 & 1.432 & 1.290 & 1.712 & 1.517 & 2.157 \\
\hline 4 & 0.485 & -1.005 & -0.314 & -0.133 & -1.781 & -0.489 & 0.683 & 1.802 & 2.237 & -1.213 \\
\hline \multirow[t]{3}{*}{ Big } & 0.224 & -0.421 & -1.803 & -4.493 & -1.792 & -2.472 & 0.278 & -0.319 & 3.504 & -1.918 \\
\hline & \multicolumn{5}{|l|}{$t(r)$} & \multicolumn{5}{|l|}{$t(h)$} \\
\hline & Low & 2 & 3 & 4 & High & Low & 2 & 3 & 4 & High \\
\hline Small & -1.609 & 0.108 & 0.503 & -2.334 & 1.961 & 3.068 & 4.063 & 2.533 & -1.440 & 2.371 \\
\hline 2 & -0.967 & -2.012 & -2.568 & -1.110 & -0.703 & 1.872 & 1.458 & 0.388 & 0.313 & -0.273 \\
\hline 3 & -2.682 & -1.296 & -0.133 & 0.042 & 0.676 & 1.808 & 1.346 & 0.574 & 2.444 & 0.131 \\
\hline 4 & -2.034 & -2.113 & -0.868 & -0.622 & -1.956 & 1.674 & 2.323 & 1.206 & 0.086 & 3.870 \\
\hline Big & -4.336 & -3.046 & -1.379 & 2.326 & -2.463 & 0.376 & -2.634 & 0.405 & 2.391 & 3.086 \\
\hline
\end{tabular}


Table 6 (continued)

\begin{tabular}{|c|c|c|c|c|c|c|c|c|c|c|}
\hline \multicolumn{11}{|c|}{ Panel D MC_TAM } \\
\hline & \multicolumn{5}{|l|}{$a$} & \multicolumn{5}{|l|}{$b$} \\
\hline & Low & 2 & 3 & 4 & High & Low & 2 & 3 & 4 & High \\
\hline Small & 0.000 & 0.011 & 0.010 & 0.005 & -0.002 & 1.138 & 0.691 & 0.912 & 1.002 & 1.006 \\
\hline 2 & 0.002 & 0.005 & 0.002 & 0.000 & -0.001 & 1.027 & 0.944 & 0.954 & 0.969 & 0.938 \\
\hline 3 & -0.006 & 0.001 & 0.007 & -0.003 & 0.002 & 1.079 & 0.995 & 0.995 & 1.142 & 0.905 \\
\hline 4 & 0.003 & 0.000 & 0.006 & 0.004 & 0.008 & 1.077 & 0.796 & 0.873 & 0.912 & 0.976 \\
\hline \multirow[t]{3}{*}{ Big } & 0.007 & 0.003 & 0.002 & 0.009 & 0.004 & 0.864 & 1.212 & 1.048 & 0.969 & 1.011 \\
\hline & \multicolumn{5}{|l|}{$S$} & \multicolumn{5}{|l|}{$L$} \\
\hline & Low & 2 & 3 & 4 & High & Low & 2 & 3 & 4 & High \\
\hline Small & 1.229 & 0.504 & 1.769 & 1.292 & 1.114 & 0.402 & 0.895 & 0.237 & 0.169 & 0.407 \\
\hline 2 & 0.464 & 0.686 & 0.412 & 0.858 & 0.314 & 0.512 & 0.136 & 0.227 & 0.478 & 0.228 \\
\hline 3 & 0.724 & 0.316 & 0.207 & 0.184 & 0.046 & 0.174 & 0.061 & -0.068 & 0.316 & 0.304 \\
\hline 4 & 0.181 & 0.094 & 0.019 & -0.228 & 0.333 & -0.060 & 0.339 & 0.262 & 0.379 & -0.103 \\
\hline \multirow[t]{3}{*}{ Big } & -0.416 & -0.250 & -0.039 & -0.205 & 0.087 & -0.146 & -0.068 & 0.087 & 0.043 & -0.372 \\
\hline & \multicolumn{5}{|l|}{$R$} & \multicolumn{5}{|l|}{$h$} \\
\hline & Low & 2 & 3 & 4 & High & Low & 2 & 3 & 4 & High \\
\hline Small & 0.361 & 0.305 & -0.967 & -0.240 & 0.022 & 0.934 & 0.958 & -0.791 & -0.316 & -0.585 \\
\hline 2 & -0.079 & -0.375 & -0.115 & 0.035 & -0.127 & 0.867 & 0.278 & 0.067 & -0.237 & -0.067 \\
\hline 3 & -0.009 & -0.265 & -0.183 & -0.115 & -0.114 & 0.781 & 0.167 & 0.223 & -0.129 & -0.041 \\
\hline 4 & -0.332 & -0.039 & -0.041 & -0.193 & -0.138 & 0.501 & 0.191 & 0.166 & -0.087 & -0.375 \\
\hline \multirow[t]{3}{*}{ Big } & -0.354 & -0.090 & -0.013 & -0.172 & -0.312 & 0.564 & 0.488 & 0.185 & -0.070 & -0.152 \\
\hline & \multicolumn{5}{|l|}{$t(a)$} & \multicolumn{5}{|l|}{$t(b)$} \\
\hline & Low & 2 & 3 & 4 & High & Low & 2 & 3 & 4 & High \\
\hline Small & 0.092 & 2.066 & 1.032 & 1.112 & -0.372 & 17.528 & 9.182 & 6.493 & 14.802 & 16.266 \\
\hline 2 & 0.380 & 1.141 & 0.463 & -0.116 & -0.137 & 14.907 & 15.710 & 15.380 & 15.890 & 15.869 \\
\hline 3 & -1.340 & 0.159 & 1.693 & -0.599 & 0.682 & 17.380 & 16.959 & 17.466 & 18.046 & 17.422 \\
\hline 4 & 0.791 & -0.107 & 1.422 & 1.201 & 2.443 & 17.291 & 14.824 & 14.429 & 17.864 & 20.151 \\
\hline \multirow[t]{3}{*}{ Big } & 1.519 & 0.750 & 0.714 & 3.091 & 1.448 & 13.468 & 24.320 & 21.128 & 24.206 & 26.912 \\
\hline & \multicolumn{5}{|l|}{$t(s)$} & \multicolumn{5}{|l|}{$t(l)$} \\
\hline & Low & 2 & 3 & 4 & High & Low & 2 & 3 & 4 & High \\
\hline Small & 8.407 & 2.976 & 5.592 & 8.473 & 7.997 & 2.457 & 4.721 & 0.669 & 0.990 & 2.613 \\
\hline 2 & 2.991 & 5.069 & 2.951 & 6.245 & 2.359 & 2.949 & 0.902 & 1.452 & 3.109 & 1.529 \\
\hline 3 & 5.177 & 2.389 & 1.612 & 1.291 & 0.392 & 1.112 & 0.414 & -0.476 & 1.982 & 2.324 \\
\hline 4 & 1.292 & 0.779 & 0.142 & -1.984 & 3.055 & -0.383 & 2.507 & 1.719 & 2.943 & -0.841 \\
\hline \multirow[t]{3}{*}{ Big } & -2.883 & -2.227 & -0.350 & -2.274 & 1.029 & -0.904 & -0.543 & 0.700 & 0.427 & -3.934 \\
\hline & \multicolumn{5}{|l|}{$t(r)$} & \multicolumn{5}{|l|}{$t(h)$} \\
\hline & Low & 2 & 3 & 4 & High & Low & 2 & 3 & 4 & High \\
\hline Small & 2.059 & 1.502 & -2.550 & -1.315 & 0.130 & 6.278 & 5.552 & -2.457 & -2.035 & -4.129 \\
\hline 2 & -0.426 & -2.312 & -0.687 & 0.213 & -0.796 & 5.487 & 2.017 & 0.470 & -1.696 & -0.493 \\
\hline 3 & -0.051 & -1.673 & -1.192 & -0.675 & -0.810 & 5.489 & 1.241 & 1.712 & -0.889 & -0.345 \\
\hline 4 & -1.976 & -0.271 & -0.251 & -1.396 & -1.057 & 3.513 & 1.555 & 1.199 & -0.742 & -3.381 \\
\hline Big & -2.042 & -0.666 & -0.095 & -1.591 & -3.074 & 3.841 & 4.268 & 1.631 & -0.761 & -1.768 \\
\hline
\end{tabular}


Table 6 (continued)

\begin{tabular}{|c|c|c|c|c|c|c|c|c|c|c|}
\hline \multicolumn{11}{|c|}{ Panel E $R^{2}$} \\
\hline & \multicolumn{5}{|c|}{ MC_PB } & \multicolumn{5}{|c|}{ MC_ROE } \\
\hline & Low & 2 & 3 & 4 & High & Low & 2 & 3 & 4 & High \\
\hline Small & 0.766 & 0.755 & 0.768 & 0.791 & 0.777 & 0.767 & 0.376 & 0.736 & 0.770 & 0.748 \\
\hline 2 & 0.535 & 0.742 & 0.765 & 0.795 & 0.794 & 0.768 & 0.787 & 0.759 & 0.756 & 0.767 \\
\hline 3 & 0.824 & 0.832 & 0.749 & 0.821 & 0.797 & 0.784 & 0.811 & 0.794 & 0.777 & 0.833 \\
\hline 4 & 0.855 & 0.785 & 0.847 & 0.828 & 0.782 & 0.803 & 0.795 & 0.775 & 0.752 & 0.802 \\
\hline \multirow[t]{3}{*}{ Big } & 0.876 & 0.886 & 0.908 & 0.880 & 0.857 & 0.883 & 0.885 & 0.886 & 0.844 & 0.830 \\
\hline & \multicolumn{5}{|c|}{ MC_TA } & \multicolumn{5}{|c|}{ MC_TAM } \\
\hline & Low & 2 & 3 & 4 & High & Low & 2 & 3 & 4 & High \\
\hline Small & 0.785 & 0.798 & 0.766 & 0.370 & 0.732 & 0.806 & 0.677 & 0.448 & 0.699 & 0.719 \\
\hline 2 & 0.801 & 0.750 & 0.797 & 0.753 & 0.719 & 0.790 & 0.769 & 0.724 & 0.732 & 0.732 \\
\hline 3 & 0.841 & 0.761 & 0.843 & 0.819 & 0.680 & 0.798 & 0.766 & 0.762 & 0.781 & 0.784 \\
\hline 4 & 0.740 & 0.821 & 0.803 & 0.823 & 0.777 & 0.788 & 0.740 & 0.714 & 0.815 & 0.770 \\
\hline Big & 0.822 & 0.839 & 0.902 & 0.890 & 0.868 & 0.730 & 0.872 & 0.826 & 0.866 & 0.866 \\
\hline
\end{tabular}

MC_PB: Two portfolios found to be significant with average alpha value of 0.0040 and 80.1 average $R$-square (\%). Market and value slopes found to be highly positive; here, size slopes become positive for big-size portfolio (which was not in case with Fama-French (2015) five-factor regression results). Similar to Fama-French (2015) in column one with low-value stocks, the coefficient of profitability (RMW) factor have positive slopes for microcaps whereas it changes its sign on increase of size. This indicates that first portfolio with combination of low value and microcaps is much dominated by the low-value stocks with aggressive investment and marginal profitability but yields higher returns than other portfolios as shown in Table 3. The biggest problem that lies with Fama-French (2015) is microcaps due to high negative profitability (RMW) and investment (CMA) factor but it is well captured by modified five-factor model with human asset as these factors become more positive.

MC_ROE: Three portfolios found to be significant with average alpha value of 0.0043 and 77.9 average $R$-square (\%). Profitability (RMW) changes its sign on moving from low ROE stock portfolios to the high ROE stock portfolios but not investment (CMA) coefficient as in case with Fama-French (2015) five-factor regressions. Biggest problem with Fama-French (2015) is that first portfolio with low profitability microcaps is dominated by firm whose stocks behave like unprofitable firms with aggressive investment due to high positive investment (CMA) and less negative profitability (RMW) coefficient which is well captured by the modified five-factor model with human asset as an addition of human asset reduces high positive investment factor.

MC_TA: Three portfolios found to be significant with average alpha value of 0.0043 and 78 average $R$-square (\%). Here also like previous case high positive investment (CMA) and less negative profitability (RMW) coefficient for microcap portfolio indicate that first portfolio with low profitability microcaps is dominated by firm whose 
Table 7 Summary of the factor regressions

\begin{tabular}{|c|c|c|c|c|c|c|c|c|}
\hline \multirow[t]{2}{*}{ Model } & \multicolumn{4}{|c|}{ No of significant intercepts } & \multicolumn{4}{|c|}{ R-Square (\%) } \\
\hline & MC_PB & MC_ROE & MC_TA & MC_TAM & MC_PB & MC_ROE & MC_TA & MC_TAM \\
\hline 3 Factor & 5 & 5 & 5 & 4 & 78.7 & 76.4 & 76.6 & 73.9 \\
\hline 5 Factor & 5 & 5 & 6 & 4 & 79.9 & 78.2 & 78.9 & 74.9 \\
\hline $\begin{array}{l}5 \text { Factor } \\
\text { (Human } \\
\text { Asset) }\end{array}$ & 2 & 3 & 3 & 3 & 80.1 & 77.9 & 78 & 75.9 \\
\hline
\end{tabular}

Three factor (MC_PB, MC_ROE, MC_TA and MC_TAM)
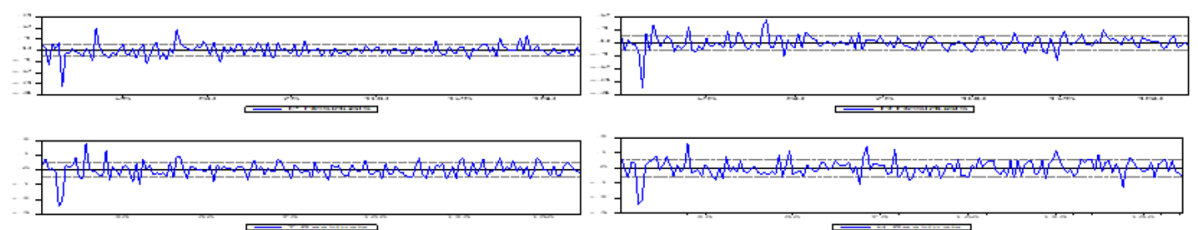

Five factor (MC_PB, MC_ROE, MC_TA and MC_TAM)
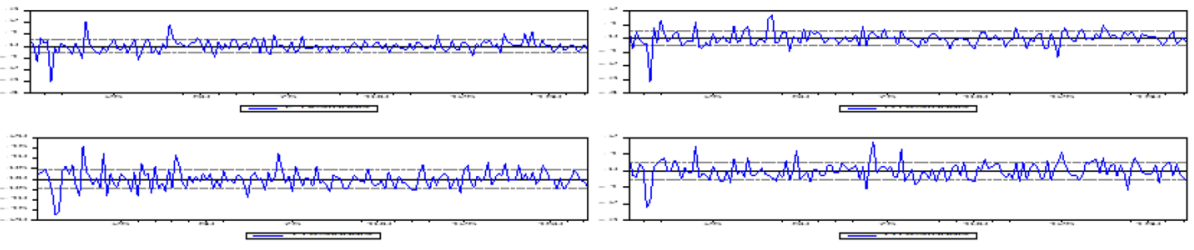

Modified Five factor with human asset (MC_PB, MC_ROE, MC_TA and MC_TAM)
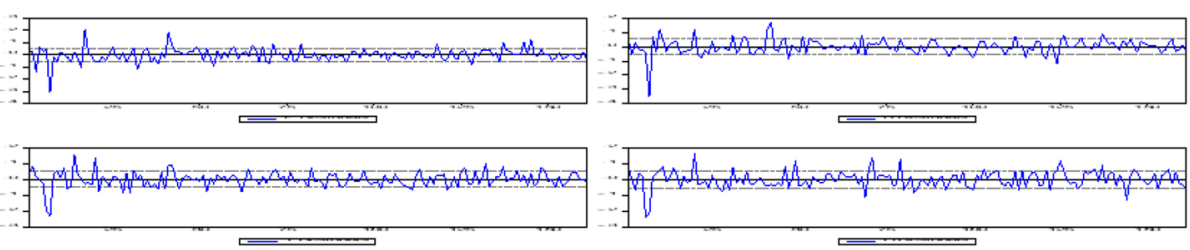

Fig. 3 Residual graphs for three-, five- and modified five-factor regressions for first Portfolio (Microcap)

stocks behave like unprofitable firms with aggressive investment and microcap not captured by the model.

MC_TAM: Three portfolios found to be significant with average alpha value of 0.0041 and 75.9 average $R$-square (\%). Similar to Fama-French three factors (1993) and Fama-French five factors (2015), microcap is well captured by the modified fivefactor model with human asset.

Study results find that modified five-factor model with human asset is robust than both Fama-French three- and five-factor models as it able to capture most of the portfolios risk return relationship. The detailed summary of all the regressions: Fama-French three-factor, Fama-French five-factor and Modified five-factor with human asset are shown in Table 7. 


\section{Residual graphs}

Then residual graphs of first Portfolio with microcaps of all regressions: Fama-French three-factor, Fama-French five-factor and Modified five-factor with human asset are shown in Fig. 3. Residual more closer to zero said to be the best model fit or model is able to capture maximum risk return relationship. Residual graphs of Fama-French three-factor and Fama-French five-factor do not show much of difference but high peaks are reduced in case of Modified five-factor with human asset that justifies superiority of the later.

Though asset pricing results and residual graphs confirm the superiority of Modified five-factor with human asset over both Fama-French three-factor and Fama-French five-factor models but it will be well justified if it passes model performance test.

\subsection{Model performance test}

The study uses very prominent asset pricing model test designed by Gibbons et al. (1989). The GRS test results are shown in Table 8 for all the factor models used in the study. GRS test rejects all the Fama-French three-factor regressions except for MC_ TAM and study findings are in line with Fama-French (2015) findings and do not support Connor and Sehgal (2003) findings in Indian context. GRS test also rejects three of the Fama-French five-factor model (2015) for MC_PB, MC_ROE \& MC_TA crosses as similar to Fama-French (2015) findings in US context. All of the Modified five-factor models with human asset except MC_TA cross and one Fama-French fivefactor model for MC_TAM crosses pass the GRS test. Further, lower value of GRS test $F$-Statistics once again confirms the superiority of Modified five-factor model with human asset over both Fama-French three-factor and Fama-French five-factor models.

Table 8 Summary of GRS test results for all the Factor models

\begin{tabular}{lllll}
\hline $\begin{array}{l}\text { Fama-French three- } \\
\text { factor }\end{array}$ & GRS F-statistics & $\boldsymbol{p}$ value & $\begin{array}{l}\text { Average absolute } \\
\text { alpha value }\end{array}$ & Average $\boldsymbol{R}^{\mathbf{2}}$ (\%) \\
\hline MC_PB $^{*}$ & 1.754 & 0.026 & 0.003 & 78.7 \\
MC_ROE* $^{\text {MC_TA* }}$ & 2.303 & 0.001 & 0.004 & 76.4 \\
MC_TAM & 1.673 & 0.045 & 0.003 & 76.6 \\
Fama-French five-factor & 1.227 & 0.228 & 0.003 & 73.9 \\
MC_PB* & 1.640 & & & 79.9 \\
MC_ROE* & 1.766 & 0.039 & 0.004 & 78.2 \\
MC_TA* & 1.594 & 0.021 & 0.004 & 78.9 \\
MC_TAM & 1.475 & 0.050 & 0.004 & 74.9 \\
Modified five-factor with human asset & 0.084 & 0.004 & 80.1 \\
MC_PB & 1.514 & & & 77.9 \\
MC_ROE & 1.532 & 0.054 & 0.004 & 78 \\
MC_TA* & 1.589 & 0.052 & 0.004 & 75.9 \\
MC_TAM & 1.497 & 0.050 & 0.004 & \\
\hline
\end{tabular}

*Significant @ 5\% level 


\section{Conclusion}

Both size and value effects are dominating in the portfolios average mean excess return patterns in Indian context. The portfolio average mean excess return with microcap stocks outperformed the other portfolios for every cross as similar to Fama-French $(1993,2015)$ findings in US context. The study also finds that portfolios formed based on MC_ROE, MC_TA and MC_TAM cross show complex return patterns as portfolios as average mean excess return patterns frequently change its slope but in spite of that size effect is consistent for all crosses. For all the crosses except MC_TAM portfolio with microcaps are not captured by the Fama-French (1993) three-factor model, the findings are in line with Aharoni et al. (2013) and Fama-French (2015) studies. Similarly all the crosses except MC_TA \& MC_TAM portfolio with microcaps are not captured by the Fama-French (2015) fivefactor model. Moreover study results find that there is no much significant difference lying between the results obtained from Fama-French three- and five-factor models. Traditionally, investment in human asset considers as the cost to the company and not as the investment Petty and Guthrie (2000). But significant number of studies by Bontis (2003) and Wright et al. (2001) argued that human asset should be considered as the investment of the firm rather than expenses in today's knowledge-based economy where human asset has greater importance in determining the value of the firm. So the present study defined a new factor of investment with human asset (CvMAv) instead of Fama-French (2015) investment (CMA) factor which generally do not consider human investment. Then, study performs regressions using modified five-factor model with market (Rm), size (SMB), value (LMH), profitability (RMW), and investment with human asset (CvMAv). The study empirically finds that modified five-factor model is robust than both Fama-French three- and five-factor models. The main problem with the modified five-factor model with human asset is the microcap with conservative investment (including human investment) stocks whose returns behave like that low-value unprofitable firms. Further microcap portfolio's (first portfolio) regression residuals are plotted using residual graphs to check the ability of various factor models that are used in the study for capturing risk return relationships. The residual graphs show more number of higher peaks in case of Fama-French three- and five-factor models' regression residuals than modified five-factor model. Residual graphs further confirm modified five-factor model is able to capture more risk return relationship. Finally study uses GRS test, a more model specific test to evaluate the performance of the various factor models. All of the Fama-French (1993) three-factor except MC_TAM cross and three Fama-French (2015) five-factor models are easily rejected by the GRS test. Whereas all modified five-factor model except MC_TA cross and one Fama-French (2015) five-factor models pass the GRS test. Further the lower values of GRS test $F$-Statistics of modified five-factor model justifies supremacy over both Fama-French three-factor and Fama-French five-factor models.

The study results clearly justify the importance of human asset in firm valuation and ignoring human asset may lead to serious issues. Investors those who are interested to gain from investment premium should consider human asset investment by the firms in making investment decision. Moreover considering human asset investment in valuation of the firms by the investors will have more informational advantages over others. Investors those whose investment patterns are based on size may gain abnormal returns by investing in the microcaps. Study finds that human asset plays a vital role in predicting returns and this has significant implications on public policy content. 


\section{Acknowledgements}

Not applicable.

\section{Authors' contributions}

Both authors read and approved the final manuscript.

\section{Funding}

Not applicable

\section{Availability of data and materials}

Data will be available only on reasonable request from the authors.

Competing interests

The authors declare that they have no competing interests.

\section{Author details}

${ }^{1}$ Department for Finance, St. Petersburg School of Economics and Management, National Research University Higher School of Economics, Kantemirovskaya St. 3A, Saint Petersburg 194100, Russia. ${ }^{2}$ Finance and Credit Department, Faculty of Economics, People's Friendship University of Russia (RUDN University), Miklukho-Maklaya str.6, Moscow 117198, Russia.

Received: 22 January 2020 Revised: 17 March 2020 Accepted: 29 June 2020

Published online: 07 July 2020

\section{References}

Aharoni G, Grundy B, Zeng Q (2013) Stock returns and the Miller Modigliani valuation formula: revisiting the Fama French analysis. J Financ Econ 110(2):347-357

Balakrishnan A, Maiti M (2017) Dynamics of size and value factors in stock returns: evidence from India. Indian J Financ $11(6): 21-35$

Banz RW (1981) The relationship between return and market value of common stocks. J Financ Econ 9(1):3-18

Basu S (1983) The relationship between earnings'yield, market value and return for NYSE common stocks: further evidence. J Financ Econ 12(1):129-156

Bell B, Machin SJ (2016) Minimum wages and firm value. https://www.econstor.eu/bitstream/10419/142353/1/dp991 4.pdf. Accessed 5 May 2017

Bhandari LC (1988) Debt/equity ratio and expected common stock returns: empirical evidence. J Financ 43(2):507-528

Black F (1972) Capital Market Equilibrium with Restricted Borrowing. J Bus 45:445-455

Bontis N (2003) Intellectual capital disclosure in Canadian corporations. J Hum Resour Cost Account 7(1):9-20

Bontis N, Keow WC, Richardson S (2000) Intellectual capital and business performance in Malaysian industries. J Intell Cap 1(1):85-100

Campbell JY (1992) Intertemporal asset pricing without consumption data (No. w3989). National Bureau of Economic Research

Campbell JY (1996) Understanding risk and return. J Polit Econ 104(2):298-345

Chen M-C, Cheng S-J, Hwang Y (2005) An empirical investigation of the relationship between intellectual capital and firms' market value and financial performance. J Intell Cap 6(2):159-176

Chiah M, Chai D, Zhong A, Li S (2016) A Better Model? An Empirical Investigation of the Fama-French Five-factor Model in Australia. Int Rev Financ 16(4):595-638

Clarke C (2016) The level, slope and curve factor model for stocks. https://www.aeaweb.org/conference/2016/retrieve. php?pdfid=1266. Accessed 5 May 2017

Cohen RB, Gompers PA, Vuolteenaho T (2002) Who underreacts to cash-flow news? Evidence from trading between individuals and institutions. J Financ Econ 66(2):409-462

Collins D, Edward M, Weiss I (1997) Changes in the value-relevance of earnings and book values over the past forty years. J Account Econ 24:39-67

Connor G, Sehgal S (2003) Tests of the Fama and French model in India. Decision 30(20):1-20

Crook R, Todd S, Combs J, Woehr D, Ketchen D (2011) Does human asset matter? A meta-analysis of the relationship between human asset and firm performance. J Appl Psychol 96(3):443-456

Draca M, Machin S, Van Reenen J (2011) Minimum wages and firm profitability. Am Econ J Appl Econ 3(1):129-151

Edmans A (2011) Does the stock market fully value intangibles? Employee satisfaction and equity prices. J Financ Econ 101(3):621-640

Fairfield PM, Whisenant JS, Yohn TL (2003) Accrued earnings and growth: implications for future profitability and market mispricing. Account Rev 78(1):353-371

Fama EF, French KR (1993) Common risk factors in the returns on stocks and bonds. J Financ Econ 33(1):3-56

Fama EF, French KR (2015) A five-factor asset pricing model. J Financ Econ 116(1):1-22

Fama EF, MacBeth JD (1973) Risk, return, and equilibrium: empirical tests. J Polit Econ 81(3):607-636

Fama EF, Schwert GW (1977) Human asset and capital market equilibrium. J Financ Econ 4(1):95-125

Gibbons MR (1982) Multivariate tests of financial models: a new approach. J Financ Econometr 10(1):3-27

Gibbons MR, Ross SA, Shanken J (1989) A test of the efficiency of a given portfolio. Econometr J Econometr Soc 57(5):1121-1152

Hansson B (2004) Human asset and stock returns: is the value premium an approximation for return on human asset? J Bus Financ Account 31(3-4):333-358

Haugen RA, Baker NL (1996) Commonality in the determinants of expected stock returns. J Financ Econ 41(3):401-439

Hou K, Xue C, Zhang L (2014) A comparison of new factor models (No. w20682). National Bureau of Economic Research 
Hou K, Xue C, Zhang L (2015) Digesting anomalies: an investment approach. Rev Financ Stud 28(3):650-705 Jagannathan R, Wang Z (1996) The conditional CAPM and the cross-section of expected returns. J Financ 51 (1):3-53

Jagannathan R, Kubota K, Takehara H (1998) Relationship between labor-income risk and average return: empirical evidence from the Japanese stock market. J Bus 71(3):319-347

Lintner J (1965) The valuation of risk assets and the selection of risky investments in stock portfolios and capital budgets. Rev Econ Stat 47(1):13-37

Maiti M (2019a) A six factor asset pricing mode

Maiti M (2019b) Is idiosyncratic risk ignored in asset pricing: Sri Lankan evidence? Future Bus J 5(1):5

Maiti M (2019c) OLS versus quantile regression in extreme distributions. Contaduría y Administración 64(2):12

Maiti M (2020a) A critical review on evolution of risk factors and factor models. J Econ Surv 34(1):175-184

Maiti M (2020b) Is ESG the succeeding risk factor? J Sustain Financ Invest. https://doi.org/10.1080/20430795.2020.17233

80

Maiti M, Balakrishnan A (2018) Is human asset the sixth factor? J Econ Stud 45(4):710-737

Maiti M, Balakrishnan A (2020) Can leverage effect coexist with value effect? IIMB Manag Rev 32(1):7-23

Mossin J (1966) Equilibrium in a capital asset market. Econometr J Econometr Soc 34(4):768-783

Novy-Marx R (2013) The other side of value: the gross profitability premium. J Financ Econ 108(1):1-28

Pantzalis C, Park JC (2009) Equity market valuation of human asset and stock returns. J Bank Finance 33(9):1610-1623

Petty R, Guthrie J (2000) Intellectual capital literature review. J Intell Cap 1(2):155-176

Qin J (2002) Human-capital-adjusted capital asset pricing model. Jpn Econ Rev 53(2):182-198

Reinganum MR (1981) Misspecification of capital asset pricing: empirical anomalies based on earnings'yields and market values. J Financ Econ 9(1):19-46

Rosett JG (2001) Equity risk and the labor stock: the case of union contracts. J Account Res 39(2):337-364

Ross SA (1976) The arbitrage theory of capital asset pricing. J Econ Theory 13(3):341-360

Shanken J (1985) Multi-beta CAPM or equilibrium APT? A reply? J Financ 40(4):1189-1196

Sharpe WF (1964) Capital asset prices: a theory of market equilibrium under conditions of risk*. J Financ 19(3):425-442

Titman S, Wei KC, Xie F (2004) Capital investments and stock returns. J Financ Quant Anal 39(04):677-700

Wright PM, Dunford BB, Snell SA (2001) Human resources and the resource based view of the firm. J Manag

27(6):701-721

\section{Publisher's Note}

Springer Nature remains neutral with regard to jurisdictional claims in published maps and institutional affiliations.

\section{Submit your manuscript to a SpringerOpen ${ }^{\circ}$ journal and benefit from:}

Convenient online submission

- Rigorous peer review

- Open access: articles freely available online

- High visibility within the field

Retaining the copyright to your article

Submit your next manuscript at $\boldsymbol{s p r i n g e r o p e n . c o m ~}$ 